\title{
Strong decays of heavy-light mesons in a chiral quark model
}

\author{
Xian-Hui Zhong ${ }^{1,3} *$ and Qiang Zhao ${ }^{1,2,3} \dagger$ \\ 1) Institute of High Energy Physics, Chinese Academy of Sciences, Beijing 100049, P.R. China \\ 2) Department of Physics, University of Surrey, Guildford, GU2 7XH, United Kingdom and \\ 3) Theoretical Physics Center for Science Facilities, CAS, Beijing 100049, P.R. China
}

\begin{abstract}
We carry out a systematic study of the heavy-light meson strong decays in a chiral quark model. For the $S$-wave vectors $\left(D^{*}(2007), D^{* \pm}(2010)\right), P$-wave scalars $\left(D_{0}^{*}(2400), B_{0}^{*}(5730)\right)$ and tensors $\left(D_{2}^{*}(2460), D_{s 2}^{*}(2573)\right)$, we obtain results in good agreement with the experimental data. For the axial vectors $D_{1}(2420)$ and $D_{1}^{\prime}(2430)$, a state mixing scheme between $1^{1} P_{1}$ and $1^{3} P_{1}$ is favored with a mixing angle $\phi \simeq-(55 \pm 5)^{\circ}$, which is consistent with previous theoretical predictions. The same mixing scheme also applies to $D_{s 1}(2460)$ and $D_{s 1}(2536)$ that accounts for the narrow width of the $D_{s 1}(2536)$ and its dominant decay into $D^{*} K$. For $B_{1}(5725)$ and $B_{1}^{\prime}(5732)$, such a mixing explains well the decay width of the former but leads to an even broader $B_{1}^{\prime}(5732)$. Predictions for the strange-bottom axial vectors are also made. For the undetermined meson $D^{*}(2640)$, we find that they fit in well the radially excited state $2^{3} S_{1}$ according to its decay mode. The newly observed $D_{s J}^{*}(2860)$ strongly favors the $D$-wave excited state $1^{3} D_{3}$. For $D_{s J}^{*}(2632)$ and $D_{s J}^{*}(2690)$, we find they are difficult to fit in any $D_{s}$ excitations in that mass region, if the experimental data are accurate. Theoretical predictions for decay modes of those unobserved states as multiplets of $2 S$ and $1 D$ waves are also presented, which should be useful for further experimental search for those states.

PACS numbers: 12.39.Fe, 12.39.Jh, 13.25.Ft,13.25.Hw
\end{abstract}

\section{INTRODUCTION}

During the past several years, significant progresses have been made in the observation of the heavy-light mesons. In 2003, two new narrow charm-strange mesons $D_{s J}^{*}(2317)$ and $D_{s J}^{*}(2460)$ were observed by BaBar, CLEO and Belle [1, 2, 3, 4, 5]. Recently, BaBar reported another two new charm-strange mesons, i.e. $D_{s J}^{*}(2860)$ with a width of $(47 \pm 17) \mathrm{MeV}$ and $D_{s J}^{*}(2690)$ with a width of $(112 \pm 43) \mathrm{MeV}$ in the $D K$ decay channel [6]. Meanwhile, Belle reported a new vector state $D_{s J}^{*}(2708)$ with a width of $\left(108 \pm 23_{-31}^{+36}\right) \mathrm{MeV}$ [7]. The $D_{s J}^{*}(2690)$ and $D_{s J}^{*}(2708)$ are believed to be the same state since their masses and widths are consistent with each other. In the $B$ meson sector two narrow states $B_{1}(5725)$ and $B_{2}^{*}(5740)$ were reported by CDF [9], and are assigned as orbitally excited $B$ mesons. They were confirmed by D0 collaboration with slightly different masses [10]. CDF collaboration also reported their strange analogues, $B_{s 1}(5829)$ and $B_{s 2}^{*}(5840)$, as orbitally excited $B_{s}$ mesons [11]. The $B_{s 2}^{*}(5840)$ is also observed by D0 collaboration 12]. About the recent experimental status of the heavy-light mesons, many reviews can be found in Refs. [13, 14, 15, 16, 17, 18, 19].

To understand the nature of the heavy-light mesons, especially the newly observed states, and to establish the heavy-light meson spectroscopy, a lot of efforts have been made on both experiment and theory. For example, one can find recent discussions about the dynamics and decay properties of the heavy-light mesons given by Close and Swanson [20], Godfrey [21], and other previous analyses in Refs. [22, 23, 24, 25, 26, 27, 28, 29, 30, 31, 32, 33]. For the new observed heavy-light mesons, such as $D_{S J}^{*}(2860)$ and $D_{s J}^{*}(2690)$, various attempts on the explanation of their nature have been made $34,35,36,37,38,39,40,41,42,43,44]$. Many systematic studies are devoted to establish the $D, D_{s}, B$, and $B_{s}$ spectroscopies [45, 46, 47, 48, 49, 50], while some earlier works can be found in Refs. 22, 51|. Recent reviews of the status of the theory study of the heavy-light mesons can be found in Refs. [52, 53, 54, 55, 56, 57, 58, 59]

On the one hand, the improved experimental measurements help clarify some old questions on the spectrum. On the other hand, they also raise some new ones which need further experimental and theoretical studies [60, 61]. For instance, $D^{*}(2640)$ reported by DELPHI in $D^{*+} \pi^{+} \pi^{-}$62] as the first radial excited state still has not yet been confirmed by any other experiments. The spin-parity of the narrow $D_{1}(2420)$ also need confirmations. The status of the broad $D_{0}^{*}(2400)$ is not clear at all, its measured mass and width have too large uncertainties. For the $D_{s}$ spectroscopy, the low masses of the $D_{s J}^{*}(2317)$ and $D_{s J}^{*}(2460)$ still cannot be well explained by theory; whether they are exotic states is an open question. Theoretical predictions for the $D_{s J}^{*}(2860)$ and $D_{s J}^{*}(2690)$ are far from convergence. The narrow state $D_{s J}^{*}(2632)$ seen by SELEX Collaboration [63] cannot be naturally explained by any

\footnotetext{
* E-mail: zhongxh@ihep.ac.cn

$\dagger$ E-mail: zhaoq@ihep.ac.cn
} 
existed theory. Nevertheless, since the flavor symmetry of the heavy-light mesons is badly broken, mixture of states with the same $J^{P}$ may occur. This will add further complexities into the meson spectrum and further theoretical investigations are needed.

In this work, we make a systematic study of the strong decays of heavy-light mesons in a chiral quark model. In the heavy-quark infinite mass limit, the flavor symmetry does no longer exist in the heavy-light mesons, which allows us to describe the initial and final $D, D_{s}, B$, and $B_{s}$ mesons in a nonrelativistic framework self-consistently. The meson decay will proceed through a single-quark transition by the emission of a pseudoscalar meson. An effective chiral Lagrangian is then introduced to account for the quark-meson coupling. Since the quark-meson coupling is invariant under the chiral transformation, some of the low-energy properties of QCD are retained. This approach is similar to that used in Refs. [22, 23], except that the two constants in the decay amplitudes of Refs. 222, 23] are replaced by two energy-dependent factors deduced from the chiral Lagrangian in our model.

The chiral quark model approach has been well developed and widely applied to meson photoproduction reactions [64, 65, 66, 67, 68, 69, 70, 71, 72]. Its recent extension to describe the process of $\pi N$ scattering and investigate the strong decays of charmed baryons also turns out to be successful and inspiring [73, 74].

The paper is organized as follows. In the subsequent section, the heavy-light meson in the quark model is outlined. Then, the non-relativistic quark-meson couplings are given in Sec. III The decay amplitudes are deduced in Sec. IV] We present our calculations and discussions in Sec. V. Finally, a summary is given in Sec. VI

\section{MESON SPECTROSCOPY}

\section{A. Harmonic oscillator states}

For a heavy-light $\bar{Q} q$ system consisting light quark 1 and heavy quark 2 with masses $m_{1}$ and $m_{2}$, respectively, its eigen-states are conventionally generated by a harmonic oscillator potential

$$
\mathcal{H}=\frac{1}{2 m_{1}} \mathbf{p}_{1}^{2}+\frac{1}{2 m_{2}} \mathbf{p}_{2}^{2}+\frac{3}{2} K\left(\mathbf{r}_{1}-\mathbf{r}_{2}\right)^{2},
$$

where vectors $\mathbf{r}_{j}$ and $\mathbf{p}_{j}$ are the coordinate and momentum for the $j$-th quark in the meson rest frame, and $K$ describes the oscillator potential strength which is independent of the flavor quantum number. One defines the Jacobi coordinates to eliminate the c.m. variables:

$$
\begin{aligned}
\mathbf{r} & =\mathbf{r}_{1}-\mathbf{r}_{2}, \\
\mathbf{R}_{c . m .} & =\frac{m_{1} \mathbf{r}_{1}+m_{2} \mathbf{r}_{2}}{m_{1}+m_{2}} .
\end{aligned}
$$

With the above relations (2 3 ), the oscillator hamiltonian (1) is reduced to

$$
\mathcal{H}=\frac{P_{c m}^{2}}{2 M}+\frac{1}{2 \mu} \mathbf{p}^{2}+\frac{3}{2} K r^{2} .
$$

where

$$
\mathbf{p}=\mu \dot{\mathbf{r}}, \quad \mathbf{P}_{c . m .}=M \dot{\mathbf{R}}_{c . m .},
$$

with

$$
M=m_{1}+m_{2}, \quad \mu=\frac{m_{1} m_{2}}{m_{1}+m_{2}} .
$$

From Eqs.(2 (3) and (5), the coordinate $\mathbf{r}_{j}$ can be expressed as functions of the Jacobi coordinate $\mathbf{r}$ :

$$
\begin{aligned}
& \mathbf{r}_{1}=\mathbf{R}_{c . m .}+\frac{\mu}{m_{1}} \mathbf{r}, \\
& \mathbf{r}_{2}=\mathbf{R}_{c . m .}-\frac{\mu}{m_{2}} \mathbf{r},
\end{aligned}
$$

and the momentum $\mathbf{p}_{j}$ is given by

$$
\begin{aligned}
& \mathbf{p}_{1}=\frac{m_{1}}{M} \mathbf{P}_{c . m .}+\mathbf{p} \\
& \mathbf{p}_{2}=\frac{m_{2}}{M} \mathbf{P}_{c . m .}-\mathbf{p} .
\end{aligned}
$$


Using standard notation, the principal quantum numbers of the oscillator is $N=(2 n+l)$, the energy of a state is given by

$$
E_{N}=\left(N+\frac{3}{2}\right) \omega,
$$

and the frequency of the oscillator is

$$
\omega=(3 K / \mu)^{1 / 2} .
$$

In the quark model the useful oscillator parameter is defined by

$$
\alpha^{2}=\mu \omega=\sqrt{\frac{2 m_{2}}{m_{1}+m_{2}}} \beta^{2},
$$

where $\beta$ is the often used harmonic oscillator parameter with a universal value $\beta=0.4 \mathrm{GeV}$. Then, the wave function of an oscillator is give by

$$
\psi_{l m}^{n}=R_{n l} Y_{l m} .
$$

TABLE I: The total wave function for the heavy-light mesons, denoted by $\left|n^{2 S+1} L_{J}\right\rangle$. The Clebsch-Gordan series for the spin and angular-momentum addition $\left|n^{2 S+1} L_{J}\right\rangle=\sum_{m+S_{z}=J_{z}}\left\langle L m, S S_{z} \mid J J_{z}\right\rangle \psi_{L m}^{n} \chi_{S_{z}} \Phi$ has been omitted, where $\Phi$ is the flavor wave function.

\begin{tabular}{|c|l|c|}
\hline \hline$\left|n^{2 S+1} L_{J}\right\rangle$ & $J^{P}$ & wave function \\
\hline $1^{1} S_{0}$ & $0^{-}$ & $\psi_{00}^{0} \chi^{0} \Phi$ \\
\hline $1^{3} S_{1}$ & $1^{-}$ & $\psi_{00}^{0} \chi_{S_{z}}^{1} \Phi$ \\
\hline $1^{1} P_{1}$ & $1^{+}$ & $\psi_{1 m}^{0} \chi^{0} \Phi$ \\
\hline $1^{3} P_{0}$ & $0^{+}$ & $\psi_{1 m}^{0} \chi_{S_{z}}^{1} \Phi$ \\
\hline $1^{3} P_{1}$ & $1^{+}$ & $\psi_{1 m}^{0} \chi_{S_{z}}^{1} \Phi$ \\
\hline $1^{3} P_{2}$ & $2^{+}$ & $\psi_{1 m}^{0} \chi_{S_{z}}^{1} \Phi$ \\
\hline $2^{1} S_{0}$ & $0^{-}$ & $\psi_{00}^{1} \chi^{0} \Phi$ \\
\hline $2^{3} S_{1}$ & $1^{-}$ & $\psi_{00}^{1} \chi_{S_{z}}^{1} \Phi$ \\
\hline $1^{1} D_{2}$ & $2^{-}$ & $\psi_{2 m}^{0} \chi^{0} \Phi$ \\
\hline $1^{3} D_{1}$ & $1^{-}$ & $\psi_{2 m}^{0} \chi_{S_{z}}^{1} \Phi$ \\
\hline $1^{3} D_{2}$ & $2^{-}$ & $\psi_{2 m}^{0} \chi_{S_{z}}^{1} \Phi$ \\
\hline $1^{3} D_{3}$ & $3^{-}$ & $\psi_{2 m}^{0} \chi_{S_{z}}^{1} \Phi$ \\
\hline
\end{tabular}

\section{B. Spin wave functions}

The usual spin wave functions are adopted. For the spin-0 state, it is

$$
\chi^{0}=\frac{1}{\sqrt{2}}(\uparrow \downarrow-\downarrow \uparrow)
$$

and for the spin-1 states, the wave functions are

$$
\begin{aligned}
& \chi_{1}^{1}=\uparrow \uparrow, \quad \chi_{-1}^{1}=\downarrow \downarrow, \\
& \chi_{0}^{1}=\frac{1}{\sqrt{2}}(\uparrow \downarrow+\downarrow \uparrow) .
\end{aligned}
$$

We take the heavy-quark infinite mass limit as an approximation to construct the total wave function without flavor symmetry. All the wave functions up to $1 D$ states are listed in Tab. I]

\section{THE QUARK-MESON COUPLINGS}

In the chiral quark model, the low energy quark-meson interactions are described by the effective Lagrangian [68, 70]

$$
\mathcal{L}=\bar{\psi}\left[\gamma_{\mu}\left(i \partial^{\mu}+V^{\mu}+\gamma_{5} A^{\mu}\right)-m\right] \psi+\cdots,
$$


where $V^{\mu}$ and $A^{\mu}$ correspond to vector and axial currents, respectively. They are given by

$$
\begin{aligned}
V^{\mu} & =\frac{1}{2}\left(\xi \partial^{\mu} \xi^{\dagger}+\xi^{\dagger} \partial^{\mu} \xi\right), \\
A^{\mu} & =\frac{1}{2 i}\left(\xi \partial^{\mu} \xi^{\dagger}-\xi^{\dagger} \partial^{\mu} \xi\right),
\end{aligned}
$$

with $\xi=\exp \left(i \phi_{m} / f_{m}\right)$, where $f_{m}$ is the meson decay constant. For the $S U(3)$ case, the pseudoscalar-meson octet $\phi_{m}$ can be expressed as

$$
\phi_{m}=\left(\begin{array}{ccc}
\frac{1}{\sqrt{2}} \pi^{0}+\frac{1}{\sqrt{6}} \eta & \pi^{+} & K^{+} \\
\pi^{-} & -\frac{1}{\sqrt{2}} \pi^{0}+\frac{1}{\sqrt{6}} \eta & K^{0} \\
K^{-} & \bar{K}^{0} & -\sqrt{\frac{2}{3}} \eta
\end{array}\right),
$$

and the quark field $\psi$ is given by

$$
\psi=\left(\begin{array}{l}
\psi(u) \\
\psi(d) \\
\psi(s)
\end{array}\right)
$$

From the leading order of the Lagrangian [see Eq.(17)], we obtain the standard quark-meson pseudovector coupling at tree level

$$
H_{m}=\sum_{j} \frac{1}{f_{m}} I_{j} \bar{\psi}_{j} \gamma_{\mu}^{j} \gamma_{5}^{j} \psi_{j} \partial^{\mu} \phi_{m} .
$$

where $\psi_{j}$ represents the $j$-th quark field in a hadron, and $I_{j}$ is the isospin operator to be given later.

In the quark model, the non-relativistic form of Eq. (21) is written as [68, 70, 73]

$$
\begin{aligned}
H_{m}^{n r}= & \sum_{j}\left\{\frac{\omega_{m}}{E_{f}+M_{f}} \boldsymbol{\sigma}_{j} \cdot \mathbf{P}_{f}+\frac{\omega_{m}}{E_{i}+M_{i}} \boldsymbol{\sigma}_{j} \cdot \mathbf{P}_{i}\right. \\
& \left.-\boldsymbol{\sigma}_{j} \cdot \mathbf{q}+\frac{\omega_{m}}{2 \mu_{q}} \boldsymbol{\sigma}_{j} \cdot \mathbf{p}_{j}^{\prime}\right\} I_{j} \varphi_{m}
\end{aligned}
$$

where $\boldsymbol{\sigma}_{j}$ corresponds to the Pauli spin vector of the $j$-th quark in a hadron. $\mu_{q}$ is a reduced mass given by $1 / \mu_{q}=$ $1 / m_{j}+1 / m_{j}^{\prime}$, where $m_{j}$ and $m_{j}^{\prime}$ stand for the masses of the $j$-th quark in the initial and final hadrons, respectively. For emitting a meson, we have $\varphi_{m}=\exp \left(-i \mathbf{q} \cdot \mathbf{r}_{j}\right)$, and for absorbing a meson we have $\varphi_{m}=\exp \left(i \mathbf{q} \cdot \mathbf{r}_{j}\right)$. In the above non-relativistic expansions, $\mathbf{p}_{j}^{\prime}=\mathbf{p}_{j}-\frac{m_{j}}{M} \mathbf{P}_{c . m}$. is the internal momentum for the $j$-th quark in the initial meson rest frame. $\omega_{m}$ and $\mathbf{q}$ are the energy and three-vector momentum of the light meson, respectively. The isospin operator $I_{j}$ in Eq. (21) is expressed as

$$
I_{j}= \begin{cases}a_{j}^{\dagger}(u) a_{j}(s) & \text { for } K^{+}, \\ a_{j}^{\dagger}(s) a_{j}(u) & \text { for } K^{-}, \\ a_{j}^{\dagger}(d) a_{j}(s) & \text { for } K^{0}, \\ a_{j}^{\dagger}(s) a_{j}(d) & \text { for } \bar{K}^{0}, \\ a_{j}^{\dagger}(u) a_{j}(d) & \text { for } \pi^{-}, \\ a_{j}^{\dagger}(d) a_{j}(u) & \text { for } \pi^{+}, \\ \frac{1}{\sqrt{2}}\left[a_{j}^{\dagger}(u) a_{j}(u)-a_{j}^{\dagger}(d) a_{j}(d)\right] & \text { for } \pi^{0}, \\ \cos \theta \frac{1}{\sqrt{2}}\left[a_{j}^{\dagger}(u) a_{j}(u)+a_{j}^{\dagger}(d) a_{j}(d)\right] & \\ -\sin \theta a_{j}^{\dagger}(s) a_{j}(s) & \text { for } \eta,\end{cases}
$$

where $a_{j}^{\dagger}(u, d, s)$ and $a_{j}(u, d, s)$ are the creation and annihilation operators for the $u, d$ and $s$ quarks. Generally, $\theta$ ranges from $\simeq 32^{\circ} \sim 43^{\circ}$ depending on quadratic or line mass relation applied [75]. In our convention, $\theta=45^{\circ}$ corresponds to the mixing scheme of Ref. [20]. We applied the same value in order to compared with Ref. [20]. However, we note in advance that within the commonly accepted range of $\theta$, our results do not show great sensitivities due to the relatively large uncertainties of the present experimental data. 
TABLE II: The spin-factors used in this work.

\begin{tabular}{l}
\hline$g_{10}^{z}=\left\langle\chi^{0}\left|\sigma_{1 z}\right| \chi_{0}^{1}\right\rangle=1$ \\
$g_{10}^{+}=\left\langle\chi^{0}\left|\sigma_{1}^{+}\right| \chi_{-1}^{1}\right\rangle=\sqrt{\frac{1}{2}}$ \\
$g_{10}^{-}=\left\langle\chi^{0}\left|\sigma_{1}^{-}\right| \chi_{1}^{1}\right\rangle=-\sqrt{\frac{1}{2}}$ \\
$g_{01}^{z}=\left\langle\chi_{0}^{1}\left|\sigma_{1 z}\right| \chi^{0}\right\rangle=1$ \\
$g_{01}^{+}=\left\langle\chi_{1}^{1}\left|\sigma_{1}^{+}\right| \chi^{0}\right\rangle=-\sqrt{\frac{1}{2}}$ \\
$g_{11}^{z}=\left\langle\chi_{1}^{1}\left|\sigma_{1 z}\right| \chi_{1}^{1}\right\rangle=1$ \\
$g_{11}^{+}=\left\langle\chi_{1}^{1}\left|\sigma_{1}^{+}\right| \chi_{0}^{1}\right\rangle=\sqrt{\frac{1}{2}}$
\end{tabular}

TABLE III: The decay amplitudes for $\left|n^{2 S+1} L_{J}\right\rangle \rightarrow\left|1^{1} S_{0}\right\rangle \mathbb{P} . g_{I}$ is a isospin factor which is defined by $g_{I}=\left\langle\phi_{\Sigma}\left|I_{1}\right| \phi_{\Lambda}\right\rangle$. In the Tab. IIIVII, the overall factor $F\left(q^{\prime}\right)=\exp \left(-\frac{q^{\prime 2}}{4 \alpha^{2}}\right)$, which plays the role of the decay form factor, is omitted for simplify, where $q^{\prime}=\left(\mu / m_{1}\right) q$. In the tables, we have defined $\mathcal{R} \equiv\left(\mathcal{G} q-\frac{1}{2} h q^{\prime}\right)$. Various spin-factors used in this work are listed in the Tab. II]

\begin{tabular}{|c|c|}
\hline \hline initial state & amplitude \\
\hline $1^{3} S_{1}\left(1^{-}\right)$ & $g_{I} g_{10}^{z} \mathcal{R}$ \\
\hline $1^{1} P_{1}\left(1^{+}\right)$ & forbidden \\
\hline $1^{3} P_{0}\left(0^{+}\right)$ & $i \frac{1}{\sqrt{6}} g_{I} g_{10}^{z} \mathcal{R} \frac{q^{\prime}}{\alpha}+i \frac{1}{\sqrt{6}} g_{I}\left(\sqrt{2} g_{10}^{+}+g_{10}^{z}\right) h \alpha$ \\
\hline $1^{3} P_{1}\left(1^{+}\right)$ & forbidden \\
\hline $1^{3} P_{2}\left(2^{+}\right)$ & $i \frac{1}{\sqrt{3}} g_{I} g_{10}^{z} \mathcal{R} \frac{q^{\prime}}{\alpha}$ \\
\hline $2^{1} S_{0}\left(0^{-}\right)$ & forbidden \\
\hline $2^{3} S_{1}\left(1^{-}\right)$ & $\frac{1}{\sqrt{24}} g_{I} g_{10}^{z} \mathcal{R}\left(\frac{q^{\prime}}{\alpha}\right)^{2}+\sqrt{\frac{1}{6}} g_{I} g_{10}^{z} h q^{\prime}$ \\
\hline $1^{1} D_{2}\left(2^{-}\right)$ & forbidden \\
\hline $1^{3} D_{1}\left(1^{-}\right)$ & $\frac{1}{\sqrt{30}} g_{I} g_{10}^{z} \mathcal{R}\left(\frac{q^{\prime}}{\alpha}\right)^{2}+\sqrt{\frac{3}{5}} g_{I} g_{10}^{+} h q^{\prime}$ \\
\hline $1^{3} D_{2}\left(2^{-}\right)$ & forbidden \\
\hline $1^{3} D_{3}\left(3^{-}\right)$ & $-\frac{1}{\sqrt{20}} g_{I} g_{10}^{z} \mathcal{R}\left(\frac{q^{\prime}}{\alpha}\right)^{2}$ \\
\hline
\end{tabular}

\section{STRONG DECAYS}

For a heavy-light meson $\bar{Q} q$, because the pseudoscalar mesons $\mathbb{P}$ only couple with the light quarks, the strong decay amplitudes for the process $\mathbb{M}_{i} \rightarrow \mathbb{M}_{f} \mathbb{P}$ can be written as

$$
\begin{aligned}
& \mathcal{M}\left(\mathbb{M}_{i} \rightarrow \mathbb{M}_{f} \mathbb{P}\right) \\
= & \left\langle\mathbb{M}_{f}\left|\left\{\mathcal{G} \boldsymbol{\sigma}_{1} \cdot \mathbf{q}+h \boldsymbol{\sigma}_{1} \cdot \mathbf{p}_{1}^{\prime}\right\} I_{1} e^{-i \mathbf{q} \cdot \mathbf{r}_{1}}\right| \mathbb{M}_{i}\right\rangle
\end{aligned}
$$

with

$$
\mathcal{G} \equiv-\left(\frac{\omega_{m}}{E_{f}+M_{f}}+1\right), \quad h \equiv \frac{\omega_{m}}{2 \mu_{q}}
$$

$\mathbb{M}_{i}$ and $\mathbb{M}_{f}$ are the initial and final meson wave functions, and they are listed in Tab. I] In the initial-meson-rest frame the energies and momenta of the initial mesons $\mathbb{M}_{i}$ are denoted by $\left(E_{i}, \mathbf{P}_{i}\right)$, while those of the final state mesons $\mathbb{M}_{f}$ and the emitted pseudoscalar mesons $\mathbb{P}$ are denoted by $\left(E_{f}, \mathbf{P}_{f}\right)$ and $\left(\omega_{m}, \mathbf{q}\right)$. Note that $\mathbf{P}_{i}=0$ and $\mathbf{P}_{f}=-\mathbf{q}$.

The form of Eq.(24) is similar to that of in Refs. [22, 23], except that the factors $\mathcal{G}$ and $h$ in this work have explicit dependence on the energies of final hadrons. In the calculations, we select $\mathbf{q}=q \hat{z}$, namely the meson moves 
TABLE IV: The decay amplitudes for $\left|n^{2 S+1} L_{J}\right\rangle \rightarrow\left|1^{3} S_{1}\right\rangle \mathbb{P}$.

\begin{tabular}{|c|c|c|}
\hline$\left|n^{2 S+1} L_{J}\right\rangle$ & $\overline{\overline{J_{z}}}$ & amplitude \\
\hline $1^{1} P_{1}\left(1^{+}\right)$ & $\begin{array}{c} \pm 1 \\
0\end{array}$ & $\begin{array}{c}i g_{I} g_{01}^{+} h \alpha \\
-i \frac{1}{\sqrt{2}} g_{I} g_{01}^{z} \mathcal{R} \frac{q^{\prime}}{\alpha}-i \frac{1}{\sqrt{2}} g_{I} g_{01}^{z} h \alpha\end{array}$ \\
\hline $1^{3} P_{0}\left(0^{+}\right)$ & & forbidden \\
\hline $1^{3} P_{1}\left(1^{+}\right)$ & $\begin{array}{c} \pm 1 \\
0\end{array}$ & $\begin{array}{c}i \frac{1}{2} g_{I} g_{11}^{z} \mathcal{R} \frac{q^{\prime}}{\alpha}+i \frac{1}{2} g_{I}\left(g_{11}^{z}+\sqrt{2} g_{11}^{+}\right) h \alpha \\
\sqrt{2} g_{I} g_{11}^{+} h \alpha\end{array}$ \\
\hline $1^{3} P_{2}\left(2^{+}\right)$ & $\begin{array}{c} \pm 1 \\
0\end{array}$ & $\begin{array}{c}-i \frac{1}{2} g_{I} g_{11}^{z} \mathcal{R} \frac{q^{\prime}}{\alpha} \\
0\end{array}$ \\
\hline $2^{1} S_{0}\left(0^{-}\right)$ & 0 & $\frac{1}{\sqrt{24}} g_{I} g_{10}^{z} \mathcal{R}\left(\frac{q^{\prime}}{\alpha}\right)^{2}+\sqrt{\frac{1}{6}} g_{I} g_{10}^{z} h q^{\prime}$ \\
\hline $2^{3} S_{1}\left(1^{-}\right)$ & $\begin{array}{c} \pm 1 \\
0\end{array}$ & $\pm\left\{\begin{array}{c}\frac{1}{\sqrt{24}} g_{I} g_{11}^{z} \mathcal{R}\left(\frac{q^{\prime}}{\alpha}\right)^{2} F+\sqrt{\frac{1}{6}} g_{I} g_{11}^{z} h q^{\prime} \\
0\end{array}\right.$ \\
\hline $1^{1} D_{2}\left(2^{-}\right)$ & $\begin{array}{c} \pm 1 \\
0\end{array}$ & $\begin{array}{c}\frac{1}{\sqrt{2}} g_{01}^{+} g_{I} h q^{\prime} \\
-\sqrt{\frac{1}{12}} g_{I} g_{01}^{z} \mathcal{R}\left(\frac{q^{\prime}}{\alpha}\right)^{2}-\sqrt{\frac{1}{3}} g_{I} g_{01}^{+} h q^{\prime}\end{array}$ \\
\hline $1^{3} D_{1}\left(1^{-}\right)$ & $\begin{array}{c} \pm 1 \\
0\end{array}$ & $\begin{array}{c}\mp\left[\sqrt{\frac{1}{120}} g_{I} g_{11}^{z} \mathcal{R}\left(\frac{q^{\prime}}{\alpha}\right)^{2}+\sqrt{\frac{5}{12}} g_{I} g_{11}^{+} h q^{\prime}\right] \\
0\end{array}$ \\
\hline $1^{3} D_{2}\left(2^{-}\right)$ & $\begin{array}{c} \pm 1 \\
0\end{array}$ & $\begin{array}{c}\sqrt{\frac{1}{24}} g_{I} g_{10}^{z} \mathcal{R}\left(\frac{q^{\prime}}{\alpha}\right)^{2}+\sqrt{\frac{3}{4}} g_{I} g_{11}^{+} h q^{\prime} \\
g_{11}^{+} g_{I} h q^{\prime}\end{array}$ \\
\hline $1^{3} D_{3}\left(3^{-}\right)$ & $\begin{array}{c} \pm 1 \\
0\end{array}$ & $\begin{array}{c}\mp \sqrt{\frac{1}{30}} g_{I} g_{11}^{z} \mathcal{R}\left(\frac{q^{\prime}}{\alpha}\right)^{2} \\
0\end{array}$ \\
\hline
\end{tabular}

TABLE V: The decay amplitudes for $\left|n^{2 S+1} L_{J}\right\rangle \rightarrow\left|1^{3} P_{0}\right\rangle \mathbb{P}$, where we have defined $\mathcal{W} \equiv \mathcal{G} q\left(-1+\frac{q^{\prime 2}}{4 \alpha^{2}}\right), \mathcal{S} \equiv h \alpha\left(1-\frac{q^{\prime 2}}{2 \alpha^{2}}\right)$

\begin{tabular}{|c|c|c|}
\hline \hline$\left|n{ }^{2 S+1} L_{J}\right\rangle$ & $J_{z}$ & amplitude \\
\hline $2^{1} S_{0}\left(0^{-}\right)$ & 0 & $i \frac{1}{3} g_{I} g_{01}^{z} \mathcal{W} \frac{q^{\prime}}{\alpha}-i \frac{1}{3} g_{I} g_{01}^{z} h \alpha \mathcal{A}$ \\
\hline $2^{3} S_{1}\left(1^{-}\right)$ & & forbidden \\
\hline & 0 & $i \frac{\sqrt{2}}{3} g_{I} g_{01}^{z} \mathcal{W} \frac{q^{\prime}}{\alpha}+i \frac{\sqrt{2}}{3} g_{I} g_{01}^{z} h \alpha \mathcal{A}$ \\
$1^{1} D_{2}\left(2^{-}\right)$ & \pm 1 & 0 \\
& \pm 2 & $-i \frac{\sqrt{2}}{3} g_{I} g_{01}^{+} h \alpha$ \\
\hline $1^{3} D_{1}\left(1^{-}\right)$ & & forbidden \\
\hline \multirow{2}{*}{$1^{3} D_{2}\left(2^{-}\right)$} & 0 & $-i \frac{\sqrt{6}}{3} g_{11}^{+} g_{I} \mathcal{S}$ \\
& \pm 2 & 0 \\
\hline $1^{3} D_{3}\left(3^{-}\right)$ & \pm 2 & $-i \frac{2}{3} g_{11}^{+} g_{I} h \alpha F-i \frac{\sqrt{2}}{6} g_{11}^{z} g_{I} \mathcal{S} h \alpha F+i \frac{1}{3} g_{11}^{z} g_{I} \mathcal{S}$ \\
& 0 & 0 \\
\hline
\end{tabular}

along the $z$ axial. Finally, we can work out the decay amplitudes for various process, $\mathbb{M} \rightarrow\left|1^{1} S_{0}\right\rangle \mathbb{P}, \mathbb{M} \rightarrow\left|1^{3} S_{1}\right\rangle \mathbb{P}$, $\mathbb{M} \rightarrow\left|1^{3} P_{0}\right\rangle \mathbb{P}, \mathbb{M} \rightarrow\left|1^{1} P_{1}\right\rangle \mathbb{P}$ and $\mathbb{M} \rightarrow\left|1^{3} P_{1}\right\rangle \mathbb{P}$, which are listed in Tabs. III VII respectively.

Some analytical features can be learned here. From Tab. III it shows that the decays of $1^{1} P_{1}, 1^{3} P_{1}, 2^{1} S_{0}, 1^{1} D_{2}$ and $1^{3} D_{2}$ into $\left|1^{1} S_{0}\right\rangle \mathbb{P}$ are forbidden by parity conservation. The decay amplitudes for $2^{3} S_{1}, 2^{3} P_{2}$, and $1^{3} D_{3} \rightarrow\left|1^{1} S_{0}\right\rangle \mathbb{P}$ are proportional to $\mathcal{R}$ (i.e. proportional to $q$ ), $\mathcal{R} q^{\prime} / \alpha$ and $\mathcal{R}(q / \alpha)^{2}$, respectively. This is crucial for understanding the small branching ratios for $D^{*}(2007) \rightarrow D \pi$ as we will see later.

In contrast, the decay amplitude for $1^{3} P_{0} \rightarrow\left|1^{1} S_{0}\right\rangle \mathbb{P}$ has two terms. One is proportional to $\mathcal{R} q^{\prime} / \alpha$, while the other is proportional to $\alpha$. Similarly, the decay amplitude for $2^{3} S_{1} \rightarrow\left|1^{1} S_{0}\right\rangle \mathbb{P}$ and $2^{3} D_{1} \rightarrow\left|1^{1} S_{0}\right\rangle \mathbb{P}$ also have two terms of which one is proportional to $\mathcal{R}\left(q^{\prime} / \alpha\right)^{2}$, and the other to $q^{\prime}$. This feature will have certain implications of their branching ratio rates into different $\left|1^{1} S_{0}\right\rangle \mathbb{P}$ states.

From Tab. IV it shows that decays of $1^{3} P_{0}$ into $\left|1^{1} S_{0}\right\rangle \mathbb{P}$ are forbidden. Among those three helicity amplitudes $\mathcal{M}_{ \pm}$and $\mathcal{M}_{0}$, the longitudinal one $\mathcal{M}_{0}$ vanishes for $1^{3} P_{2}, 2^{3} S_{1}, 1^{3} D_{1}$, and $1^{3} D_{3}$ into $\left|1^{1} S_{0}\right\rangle \mathbb{P}$.

From Tabs. $\mathrm{V} \mathrm{VII}$ we can see that the decays of $2^{3} S_{1}$ and $1^{3} D_{1}$ into $\left|1^{3} P_{0}\right\rangle \mathbb{P}, 2^{1} S_{0}$ and $1^{1} D_{2}$ into $\left|1^{1} P_{1}\right\rangle \mathbb{P}$, and $2^{1} S_{0}$ into $\left|1^{1} P_{1}\right\rangle \mathbb{P}$ are forbidden parity conservation. These selection rules are useful for the state classifications. 
TABLE VI: The decay amplitudes for $\left|n^{2 S+1} L_{J}\right\rangle \rightarrow\left|1^{1} P_{1}\right\rangle \mathbb{P}$.

\begin{tabular}{|c|c|c|}
\hline \hline$\left.{ }^{2 S+1} L_{J}\right\rangle$ & $\left(J_{z}^{f}, J_{z}^{i}\right)$ & amplitude \\
\hline $2^{1} S_{0}\left(0^{-}\right)$ & & forbidden \\
\hline $2^{3} S_{1}\left(1^{-}\right)$ & $\pm(1,-1)$ & $-i \sqrt{\frac{2}{3}} g_{I} g_{10}^{+} h \alpha\left(1+\frac{q^{\prime 2}}{4 \alpha^{2}}\right)$ \\
& $(0,0)$ & $-i \frac{1}{\sqrt{3}} g_{I} g_{10}^{z} \mathcal{W} \frac{q^{\prime}}{\alpha}+i \frac{1}{\sqrt{3}} g_{I} g_{10}^{z} h \alpha \mathcal{A}$ \\
\hline $1^{1} D_{2}\left(2^{-}\right)$ & & forbidden \\
\hline $1^{3} D_{1}\left(1^{-}\right)$ & $\pm(1,-1)$ & $-i \sqrt{\frac{3}{20}} g_{I} g_{10}^{z} \mathcal{G} q \frac{q^{\prime}}{\alpha}-i \sqrt{\frac{1}{30}} g_{I} g_{10}^{+} \mathcal{S}$ \\
& $(0,0)$ & $-i \sqrt{\frac{4}{15}} g_{I} g_{10}^{z} \mathcal{W} \frac{q^{\prime}}{\alpha}+i \sqrt{\frac{4}{15}} g_{I} g_{10}^{z} h \alpha \mathcal{A}$ \\
\hline \hline $1^{3} D_{2}\left(2^{-}\right)$ & $\pm(1,-1)$ & $-i \frac{1}{\sqrt{12}} g_{I} g_{10}^{z} \mathcal{R} \frac{q^{\prime}}{\alpha}+i \frac{1}{\sqrt{24}} g_{I} g_{10}^{+} h q^{\prime} \frac{q^{\prime}}{\alpha}$ \\
\hline $1^{3} D_{3}\left(3^{-}\right)$ & $\pm(1,-1)$ & $-i \sqrt{\frac{1}{30}}\left(\sqrt{2} g_{10}^{z}-g_{10}^{+}\right) g_{I} h q^{\prime} \frac{q^{\prime}}{\alpha}$ \\
& $(0,0)$ & $i \sqrt{\frac{1}{5}} g_{I}\left[g_{10}^{z} \mathcal{W} \frac{q^{\prime}}{\alpha}-\sqrt{2} g_{10}^{z} h \alpha \mathcal{A}+2 g_{10}^{+} \mathcal{S}\right]$ \\
\hline
\end{tabular}

TABLE VII: The decay amplitudes for $\left|n^{2 S+1} L_{J}\right\rangle \rightarrow\left|1^{3} P_{1}\right\rangle \mathbb{P}$.

\begin{tabular}{|c|c|c|}
\hline \hline$\left.n^{2 S+1} L_{J}\right\rangle$ & $\left(J_{z}^{f}, J_{z}^{i}\right)$ & amplitude \\
\hline $2^{1} S_{0}\left(0^{-}\right)$ & & forbidden \\
\hline $2^{3} S_{1}\left(1^{-}\right)$ & $\pm(1,-1)$ & $-i \frac{1}{\sqrt{3}} g_{I} g_{11}^{+} h \alpha\left(1+\frac{q^{\prime 2}}{4 \alpha^{2}}\right)$ \\
& $\pm(1,1)$ & $i \frac{1}{\sqrt{6}} g_{I} g_{11}^{z} \mathcal{W} \frac{q^{\prime}}{\alpha}-i \frac{1}{\sqrt{6}} g_{I} g_{11}^{z} h \alpha \mathcal{A}$ \\
\hline \multirow{2}{*}{$1^{1} D_{2}\left(2^{-}\right)$} & $\pm(0,2)$ & $\pm i g_{01}^{+} h \alpha$ \\
& $\pm(1,1)$ & $\pm i \frac{1}{\sqrt{2}} g_{01}^{+} \mathcal{S}$ \\
& $\pm(1,-1)$ & $\pm i \frac{1}{2} g_{I} g_{01}^{z}\left(\mathcal{R} \frac{q^{\prime}}{\alpha}+h \alpha\right)$ \\
\hline $1^{3} D_{1}\left(1^{-}\right)$ & $\pm(1,-1)$ & $-i \sqrt{\frac{1}{60}} g_{I} g_{11}^{+} h \alpha\left(1+\frac{q^{\prime 2}}{2 \alpha^{2}}\right)$ \\
& $\pm(1,1)$ & $-i \sqrt{\frac{1}{30}} g_{I} g_{11}^{z}\left(\mathcal{W} \frac{q^{\prime}}{\alpha}-h \alpha \mathcal{A}-\frac{3}{2} \mathcal{S}\right)$ \\
\hline \hline & $\pm(0,2)$ & $\pm i \frac{1}{\sqrt{12}} g_{I} g_{11}^{z}\left(\mathcal{R} \frac{q^{\prime}}{\alpha}+3 h \alpha\right)$ \\
$1^{3} D_{2}\left(2^{-}\right)$ & $\pm(1,-1)$ & $\pm\left[-i \sqrt{\frac{1}{3}} g_{I} g_{11}^{z} h \alpha-i \sqrt{\frac{1}{12}} g_{11}^{+} \mathcal{S}\right]$ \\
& $\pm(1,1)$ & $\pm i \sqrt{\frac{1}{6}} g_{I} g_{11}^{z}\left(\mathcal{W} \frac{q^{\prime}}{\alpha}-h \alpha \mathcal{A}-\frac{1}{2} \mathcal{S}\right)$ \\
\hline & $\pm(0,2)$ & $i \frac{1}{\sqrt{6}} g_{I} g_{11}^{z} \mathcal{R} \frac{q^{\prime}}{\alpha}$ \\
$1^{3} D_{3}\left(3^{-}\right)$ & $\pm(1,-1)$ & $-i \sqrt{\frac{1}{60}} g_{I} g_{11}^{+} h q^{\prime} \frac{q^{\prime}}{\alpha}$ \\
& $\pm(1,1)$ & $-i \sqrt{\frac{2}{15}} g_{I} g_{11}^{z}\left[\mathcal{W} \frac{q^{\prime}}{\alpha}-h \alpha \mathcal{A}+\mathcal{S}\right]$ \\
\hline
\end{tabular}

\section{CALCULATIONS AND ANALYSIS}

With the transition amplitudes, one can calculate the partial decay width with

$$
\Gamma=\left(\frac{\delta}{f_{m}}\right)^{2} \frac{\left(E_{f}+M_{f}\right)|\mathbf{q}|}{4 \pi M_{i}\left(2 J_{i}+1\right)} \sum_{J_{i z}, J_{f z}}\left|\mathcal{M}_{J_{i z}, J_{f z}}\right|^{2}
$$

where $J_{i z}$ and $J_{f z}$ stand for the third components of the total angular momenta of the initial and final heavy-light mesons, respectively. $\delta$ as a global parameter accounts for the strength of the quark-meson couplings. In the heavylight meson transitions, the flavor symmetry does not hold any more. Treating the light pseudoscalar meson as a chiral field while treating the heavy-light mesons as constitute quark system is an approximation. This will bring uncertainties to coupling vertices and form factors. Parameter $\delta$ is introduced to take into account such an effect. It has been determined in our previous study of the strong decays of the charmed baryons [74]. Here, we fix its value the same as that in Ref. [74], i.e. $\delta=0.557$.

In the calculation, the standard parameters in the quark model are adopted. For the $u, d, s, c$ and $b$ constituent quark masses we set $m_{u}=m_{d}=350 \mathrm{MeV}, m_{s}=550 \mathrm{MeV}, m_{c}=1700 \mathrm{MeV}$ and $m_{b}=5100 \mathrm{MeV}$, respectively. The decay constants for $\pi, K, \eta$ and $D$ mesons, $f_{\pi}=132 \mathrm{MeV}, f_{K}=f_{\eta}=160 \mathrm{MeV}$, and $f_{D}=226 \mathrm{MeV}$, are used. For the masses of all the heavy-light mesons the PDG values are adopted in the calculations [75].

To partly remedy the inadequate of the non-relativistic wave function as the relative momentum $q$ increases, a 
commonly used Lorentz boost factor is introduced into the decay amplitudes [67, 72, 73],

$$
\mathcal{M}(q) \rightarrow \gamma_{f} \mathcal{M}\left(\gamma_{f} q\right)
$$

where $\gamma_{f}=M_{f} / E_{f}$. In most decays, the three momentum carried by the final state mesons are relatively small, which means the non-relativistic prescription is reasonable and corrections from Lorentz boost are not drastic.

TABLE VIII: Predictions of the strong decay widths (in $\mathrm{MeV}$ ) for the heavy-light mesons. For comparison, the experimental data and some other model predictions are listed.

\begin{tabular}{|c|c|c|c|c|c|c|c|c|c|}
\hline & notation & $\overline{\text { channel }}$ & $\Gamma$ (this work) & 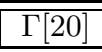 & $\overline{\Gamma[21]}$ & $\overline{\bar{\Gamma}[33]}$ & $\overline{\Gamma[31,32]}$ & $\begin{array}{l}\Gamma[27] \\
\end{array}$ & 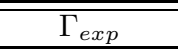 \\
\hline $\begin{array}{l}D^{*}(2007)^{0} \\
D^{*}(2010)^{+}\end{array}$ & $\left(1^{3} S_{1}\right) 1^{-}$ & $\begin{array}{l}D^{0} \pi^{0} \\
D^{0} \pi^{+} \\
D^{+} \pi^{0}\end{array}$ & $\begin{array}{l}58 \mathrm{keV} \\
77 \mathrm{keV} \\
35 \mathrm{keV}\end{array}$ & $\begin{array}{l}16 \mathrm{keV} \\
25 \mathrm{keV} \\
11 \mathrm{keV}\end{array}$ & & \begin{tabular}{|ll}
$39 \mathrm{keV}$ \\
$60 \mathrm{keV}$ \\
$27 \mathrm{keV}$
\end{tabular} & & & $\begin{array}{c}<2.1 \mathrm{MeV} \\
64 \pm 15 \mathrm{keV} \\
29 \pm 7 \mathrm{keV}\end{array}$ \\
\hline $\begin{array}{l}D_{0}^{*}(2352) \\
D_{0}^{*}(2403) \\
\end{array}$ & $\left(1^{3} P_{0}\right) 0^{+}$ & $D \pi$ & $\begin{array}{l}248 \\
266\end{array}$ & 283 & 277 & & & & $\begin{array}{l}261 \pm 50 \\
283 \pm 58\end{array}$ \\
\hline$D_{1}(2420)$ & $\begin{array}{c}\left(1^{1} P_{1}\right) 1^{+} \\
\left(P_{1}\right) 1^{+}\end{array}$ & $D^{*} \pi$ & $\begin{array}{c}84 \\
21.6 \\
\end{array}$ & 22 & 25 & & & & $25 \pm 6$ \\
\hline \begin{tabular}{|l}
$D_{1}^{\prime}(2430)$ \\
\end{tabular} & $\begin{array}{c}\left(1^{3} P_{1}\right) 1^{+} \\
\left(P_{1}^{\prime}\right) 1^{+} \\
\end{array}$ & $\overline{D^{*} \pi}$ & $\begin{array}{l}152 \\
220 \\
\end{array}$ & 272 & 244 & & & & $384 \pm 117$ \\
\hline$D_{2}^{*}(2460)^{0}$ & $\left(1^{3} P_{2}\right) 2^{+}$ & $\begin{array}{c}D \pi \\
D^{*} \pi \\
D \eta \\
\text { total } \\
\end{array}$ & $\begin{array}{l}39 \\
19 \\
0.1 \\
59 \\
\end{array}$ & $\begin{array}{c}35 \\
20 \\
0.08 \\
55 \\
\end{array}$ & $\begin{array}{r}37 \\
18 \\
55 \\
\end{array}$ & & $\begin{array}{c}13.7 \\
6.1 \\
\\
20 \\
\end{array}$ & & $43 \pm 4$ \\
\hline$\overline{D_{s 1}(2536)}$ & $\begin{array}{c}\left(1^{\mathrm{I}} P_{1}\right) 1^{+} \\
\left(P_{1}\right) 1^{+}\end{array}$ & $\overline{\overline{D^{*} K}}$ & $\begin{array}{c}59 \\
0.35\end{array}$ & 0.8 & 0.34 & & & & $<2.3$ \\
\hline \begin{tabular}{|l}
$D_{s 2}^{*}(2573)$ \\
\end{tabular} & $\left(1^{3} P_{2}\right) 2^{+}$ & $\begin{array}{c}D K \\
D^{*} K \\
D_{s} \eta \\
\text { total } \\
\end{array}$ & $\begin{array}{c}16 \\
1 \\
0.4 \\
17\end{array}$ & $\begin{array}{c}27 \\
3.1 \\
0.2 \\
30 \\
\end{array}$ & $\begin{array}{c}20 \\
1 \\
21\end{array}$ & & & & $15_{-4}^{+5}$ \\
\hline$\overline{D_{s J}^{*}(2860)}$ & $\left(1^{3} D_{3}\right) 3^{-}$ & $\begin{array}{c}D K \\
D^{*} K \\
D_{s} \eta \\
D_{s}^{*} \eta \\
D K^{*} \\
\text { total }\end{array}$ & $\begin{array}{c}27 \\
11 \\
3 \\
0.3 \\
0.4 \\
42\end{array}$ & & & & & & $48 \pm 17$ \\
\hline$\overline{\mid B_{0}^{*}(5730)}$ & $\overline{\left({ }^{3} P_{0}\right) 0^{+}}$ & $\overline{\overline{B \pi}}$ & $\overline{272}$ & & & $\overline{141}$ & 250 & & \\
\hline$B_{1}(5725)$ & $\left(P_{1}\right) 1^{+}$ & $B^{*} \pi$ & 30 & & & 20 & & & $20 \pm 12$ \\
\hline$B_{1}^{\prime}(5732)$ & $\begin{array}{c}\left(1^{3} P_{1}\right) 1^{+} \\
\left(P_{1}^{\prime}\right) 1^{+}\end{array}$ & $\begin{array}{l}B^{*} \pi \\
B^{*} \pi\end{array}$ & $\begin{array}{l}153 \\
219\end{array}$ & & & 139 & 250 & & $128 \pm 18$ \\
\hline$B_{2}^{*}(5740)$ & $\left(1^{3} P_{2}\right) 2^{+}$ & $\begin{array}{c}B \pi \\
B^{*} \pi \\
\text { total } \\
\end{array}$ & $\begin{array}{l}25 \\
22 \\
47 \\
\end{array}$ & & & $\begin{array}{l}15 \\
14 \\
29 \\
\end{array}$ & $\begin{array}{l}3.9 \\
3.4 \\
7.3 \\
\end{array}$ & $16 \pm 6$ & $22_{-6}^{+7}$ \\
\hline$B_{s 0}^{*}(5800)$ & $\overline{\left({ }^{3} P_{0}\right) 0^{+}}$ & $\overline{B B K}$ & 227 & & & & & & \\
\hline$B_{s 1}(5830)$ & $\left(P_{1}\right) 1^{+}$ & $B^{*} K$ & $0.4 \sim 1$ & & & & & $3 \pm 1$ & 1 \\
\hline$B_{s 1}^{\prime}(5830)$ & $\left(P_{1}^{\prime}\right) 1^{+}$ & $B^{*} K$ & 149 & & & & & & \\
\hline$B_{s 2}^{*}(5839)$ & $\left(1^{3} P_{2}\right) 2^{+}$ & $\begin{array}{l}B K \\
B^{*} K \\
\text { total }\end{array}$ & $\begin{array}{c}2 \\
0.12 \\
2\end{array}$ & & & & & $7 \pm 3$ & 1 \\
\hline
\end{tabular}

\section{A. Strong decays of $1 S$ states}

Due to isospin violation, $D^{*+}$ is about $3 \mathrm{MeV}$ heavier than the neutral $D^{* 0}$ [75]. This small difference leads to a kinematic forbiddance of $D^{* 0} \rightarrow D^{+} \pi^{-}$, while $D^{*+} \rightarrow D^{0} \pi^{+}$and $D^{+} \pi^{0}$ are allowed, but with a strong kinematic suppression. Nevertheless, it shows by Tab. III that the decay amplitudes of $1 S$ states are proportional to the final state momentum $q$. For the decays of $D^{* 0} \rightarrow D^{0} \pi^{0}, D^{*+} \rightarrow D^{0} \pi^{+}$and $D^{+} \pi^{0}$ of which the decay thresholds are close to the $D^{*}$ masses, it leads to further dynamic suppressions to the partial decay widths. As shown in Tab. VIII, our calculations are in remarkable agreement with the experimental data. Since $q$ is small, the form factor corrections 
from quark model are negligibly small. One would expect that the ratio $\Gamma\left(D^{0} \pi^{+}\right) / \Gamma\left(D^{+} \pi^{0}\right) \simeq 2$ is then dominated by the isospin factor $g_{I}$, which agrees well with the prediction in Ref. [20].

\section{B. Strong decays of $1 P$ states}

In the $L S$ coupling scheme, there are four $1 P$ states: ${ }^{3} P_{0},{ }^{3} P_{1},{ }^{3} P_{2}$ and ${ }^{1} P_{1}$. For ${ }^{3} P_{0}$, its transition to $\left|1^{3} S_{1}\right\rangle \mathbb{P}$ is forbidden. States of ${ }^{1} P_{1}$, and ${ }^{3} P_{1}$ can couple into $\left|1^{3} S_{1}\right\rangle \mathbb{P}$, but not $\left|1^{1} S_{0}\right\rangle \mathbb{P}$. In contrast, ${ }^{3} P_{2}$ can be coupled to both $\left|1^{3} S_{1}\right\rangle \mathbb{P}$ and $\left|1^{1} S_{0}\right\rangle \mathbb{P}$. In the decay amplitudes of ${ }^{3} P_{0},{ }^{1} P_{1}$, and ${ }^{3} P_{1}$, the term $h \alpha F$ dominates the partial decay widths, and usually their decay widths are much broader than that of ${ }^{3} P_{2}$. Between the amplitudes of the ${ }^{1} P_{1}$ and ${ }^{3} P_{1}$ decays, we approximately have:

$$
\mathcal{M}\left({ }^{1} P_{1} \rightarrow\left|1^{3} S_{1}\right\rangle \mathbb{P}\right)_{J_{z}} \simeq \frac{1}{\sqrt{2}} \mathcal{M}\left({ }^{3} P_{1} \rightarrow\left|1^{3} S_{1}\right\rangle \mathbb{P}\right)_{J_{z}}
$$

since the term $\mathcal{R} \frac{q^{\prime}}{\alpha} F$ is negligible when the decay channel threshold is close to the initial meson mass. As a consequence, the decay widths of the ${ }^{1} P_{1}$ states are narrower than those of ${ }^{3} P_{1}$.

$$
\text { 1. }{ }^{3} P_{0} \text { states }
$$

$D_{0}^{*}(2400)$ is listed in PDG [75] as a broad ${ }^{3} P_{0}$ state. Its mass values from Belle [76] and FOCUS Collaboration 77] are quite different though the FOCUS result is consistent with the potential quark model prediction of $2403 \mathrm{MeV}$ [22]. In experiment, only the $D \pi$ channel are observed since the other channels are forbidden. The term $h \alpha F$ in the amplitude, which is in proportion to the oscillator parameter $\alpha$, accounts for the broad decay width. By applying the PDG averaged mass $2352 \mathrm{MeV}$ and the FOCUS value $2403 \mathrm{MeV}$, its partial decay widths into $D \pi$ are calculated and presented in Tab. VIII] They are in good agreement with the data [75, 77].

In the $B$ meson sector, $B_{0}^{*}$ and $B_{s 0}^{*}$, as the ${ }^{3} P_{0}$ states, have not been confirmed in any experiments. The predicted mass for $B_{0}^{*}$ and $B_{s 0}^{*}$ mesons are about $5730 \mathrm{MeV}$ and $5800 \mathrm{MeV}$, respectively [48]. Their strong decays only open to $B \pi$ or $B K$. Applying the theory-predicted masses, we obtain broad decay widths for both states, i.e. $\Gamma\left(B_{0}^{*}\right)=272$ $\mathrm{MeV}$, and $\Gamma\left(B_{s 0}^{*}\right)=227 \mathrm{MeV}$, respectively. Our prediction of $\Gamma\left(B_{0}^{*}\right)=272 \mathrm{MeV}$ is compatible with the QCDSR prediction $\Gamma\left(B_{0}^{*}\right) \simeq 250 \mathrm{MeV}$ [31]. Such broad widths may explain why they have not yet been identified in experiment.

$$
\text { 2. }{ }^{3} P_{2} \text { states }
$$

In PDG, the decay width of $D_{2}^{*}(2460)^{0}$ is $\Gamma=43 \pm 4 \mathrm{MeV}$ and that of $D_{2}^{*}(2460)^{ \pm}$is $\Gamma=29 \pm 5 \mathrm{MeV}$. Since there is no obvious dynamic reason for such a significant difference, it may simply be due to experimental uncertainties. Our prediction $\Gamma=59 \mathrm{MeV}$ as a sum of the partial widths of $D \pi, D^{*} \pi$ and $D \eta$, is comparable with the data. Nevertheless, the partial width ratio

$$
R \equiv \frac{\Gamma(D \pi)}{\Gamma\left(D^{*} \pi\right)} \simeq 2.1
$$

obtained here is also in good agreement with the data $R \simeq 2.3 \pm 0.6$ 75] .

$D_{s 2}^{*}(2573)$ is assigned to be a ${ }^{3} P_{2}$ state. Its total width is $\Gamma_{\text {exp }}=15_{-4}^{+5}$ and the width ratio between $D^{*} K$ and $D K$ is $R \equiv \Gamma\left(D^{*} K\right) / \Gamma(D K)<0.33[75]$. Our predictions for the total width and ratio $R$ are

$$
\begin{aligned}
\Gamma & =17 \mathrm{MeV} \\
R & \equiv \frac{\Gamma\left(D^{*} K\right)}{\Gamma(D K)} \simeq 6 \%,
\end{aligned}
$$

which are consistent with the data.

Notice that the width of $D^{*} K, \sim 1 \mathrm{MeV}$, is one-order-of-magnitude smaller than that of $D K$. Apart from the kinematic phase space suppression, its transition amplitude also suffers dynamic suppressions since it is proportional to $\mathcal{R} q^{\prime} / \alpha$. This explains its absence in experiment. Although the decay channel $D \eta / D_{s} \eta$ is also opened for $D_{2}^{*}(2460) / D_{s 2}^{*}(2573)$, its partial width is negligibly small, i.e. $<1 \mathrm{MeV}$. 
In the $B$ meson sector, a candidate of ${ }^{3} P_{2}$ state is from CDF collaboration with mass [9].

$$
M\left(B_{2}^{*}\right)=5740 \pm 2 \pm 1 \mathrm{MeV} .
$$

D0 collaboration also observed the same state with slightly different masses, $M\left(B_{2}^{*}\right)=5746.8 \pm 2.4 \pm 1.7 \mathrm{MeV}[10]$. By assigning $B_{2}^{*}$ as a ${ }^{3} P_{2}$ state, the predicted total width as sum of $B \pi$ and $B^{*} \pi$ is

$$
\Gamma\left(B_{2}^{*}\right) \simeq 47 \mathrm{MeV}
$$

which is consistent with the CDF measurement $\Gamma\left(B_{2}^{*}\right)_{\exp } \simeq 22_{-6}^{+7} \mathrm{MeV}$. It shows that these two partial widths of $B \pi$ and $B^{*} \pi$ are comparable with each other, and the predicted width ratio is

$$
R \equiv \frac{\Gamma\left(B^{*} \pi\right)}{\Gamma\left(B^{*} \pi\right)+\Gamma(B \pi)}=0.47
$$

This is also in good agreement with the recent D0 data $R=0.475 \pm 0.095 \pm 0.069$ [10].

CDF collaboration also reported an observation of $B_{2}^{*}$ 's strange analogue $B_{s 2}^{*}$ [11], of which the mass is

$$
M\left(B_{s 2}^{*}\right)=5840 \pm 1 \mathrm{MeV} .
$$

With this mass, we obtain its partial decay widths, $\Gamma\left(B^{*} K\right)=0.12 \mathrm{MeV}$ and $\Gamma(B K)=2 \mathrm{MeV}$, respectively. This gives its strong decay width and width ratio between $B^{*} K$ and $B K$ :

$$
\Gamma\left(B_{s 2}^{*}\right) \simeq 2 \mathrm{MeV}, R \equiv \frac{\Gamma\left(B^{*} K\right)}{\Gamma(B K)} \simeq 6 \% .
$$

The decay width is in good agreement with the data $\Gamma\left(B_{s 2}^{*}\right)_{\exp } \sim 1 \mathrm{MeV}$ [82]. It also shows that the partial width of $B^{*} K$ channel is negligible small, and will evade from observations in experiment. But a measurement of $\Gamma(B K)$ with improved statistics should be very interesting.

\section{The mixed states}

The $D_{1}(2420)$ and $D_{1}^{\prime}(2430)$ listed in PDG [75] correspond to a narrow and broad state, respectively. Their two body pionic decays are only seen in $D^{*} \pi$. If they are pure $P$ wave states, they should be correspondent to ${ }^{1} P_{1}$ and ${ }^{3} P_{1}$. The calculated decay widths by assigning them as ${ }^{1} P_{1}$ and ${ }^{3} P_{1}$, are listed in Tab. VIII. It shows that $D_{1}(2420)$ as a pure ${ }^{1} P_{1}$ state, its decay width is overestimated by about an order, while $D_{1}^{\prime}(2430)$ as a pure ${ }^{3} P_{1}$ state, its decay width is underestimated by about a factor of 2 . Similarly large discrepancies are also found if one simply exchanges the assignments. Thus, the pure ${ }^{1} P_{1}$ and ${ }^{3} P_{1}$ scenario cannot explain the nature of $D_{1}(2420)$ and $D_{1}^{\prime}(2430)$.

Since the heavy-light mesons are not charge conjugation eigenstates, state mixing between spin $\mathbf{S}=0$ and $\mathbf{S}=1$ states with the same $J^{P}$ can occur. The physical states with $J^{P}=1^{+}$would be given by

$$
\begin{aligned}
\left|P_{1}^{\prime}\right\rangle & =+\cos (\phi)\left|{ }^{1} P_{1}\right\rangle+\sin (\phi)\left|{ }^{3} P_{1}\right\rangle, \\
\left|P_{1}\right\rangle & \left.=-\sin (\phi)\left|{ }^{1} P_{1}\right\rangle+\left.\cos (\phi)\right|^{3} P_{1}\right\rangle .
\end{aligned}
$$

Our present knowledge about the $D_{1}(2420)$ and $D_{1}^{\prime}(2430)$ mixing is still limited. The determination of the mixing angle is correlated with quark potential, and masses of the states [23]. An analysis by Ref. [20] suggests that a mixed state dominated by $S$-wave decay will have a broad width, and the $D$-wave-dominant decay will have a narrow one. By assuming that the heavy quark spin-orbit interaction is positive, this leads to an assignment of $D_{1}^{\prime}(2430)$ and $D_{1}(2420)$ as a mixed $\left|P_{1}^{\prime}\right\rangle$ and $\left|P_{1}\right\rangle$, respectively, with a negative mixing angle $\phi=-54.7^{\circ}$. However, this will lead to that the mass of $D_{1}$ is heavier that of the $D_{1}^{\prime}$ for which the present experimental precision seem unable to rule out such a possibility [75]. An additional piece of information supporting such a scenario is that a positive spin-orbit interaction will lead to a heavier $2^{+}$state than $0^{+}$which indeed agrees with experiment [20].

In our calculation, we plot the pionic decay widths of the mixed states $\left|P_{1}^{\prime}\right\rangle$ and $\left|P_{1}\right\rangle$ as functions of $\phi$ in Fig. 1. By looking for the best description of the experimental data, we determine the optimal mixing angle. It shows that with $\phi=-(55 \pm 5)^{\circ}, D_{1}(2420)$, as the $\left|P_{1}\right\rangle$ mixed state, has a narrow decay width of $\Gamma \simeq 22 \mathrm{MeV}$. This value agrees well with the experimental data (see Tab. VIII). Our prediction for the width of $D_{1}^{\prime}(2430)$ as a $\left|P_{1}^{\prime}\right\rangle$ broad state is 

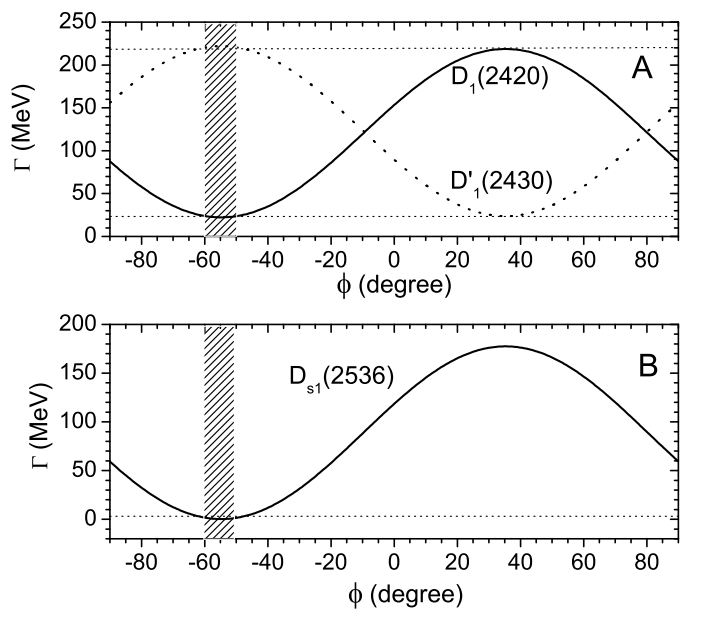

FIG. 1: The decay widths of $D_{1}(2420), D_{1}(2430)$ and $D_{s 1}(2536)$ as functions of the mixing angle $\phi$.

$\Gamma \simeq 217 \mathrm{MeV}$, which also agrees with the data 75$]$. Note that there are still large uncertainties with the $D_{1}^{\prime}(2430)$ measurements, and further experimental investigation is needed.

Such a mixing scenario may occur within the $D_{s 1}$ states, which leads to $D_{s 1}(2460)$ and $D_{s 1}(2536)$ as the mixed $\left|P_{1}^{\prime}\right\rangle$ and $\left|P_{1}\right\rangle$, respectively. Note that $D_{s 1}(2460)$ has a relatively light mass which is below the $D^{*} K$ threshold, and also slightly below the $D K$ threshold. Therefore, its strong decay is nearly forbidden, which makes it a narrow state. On the other hand, $D_{s 1}(2536)$, as a $\left|P_{1}\right\rangle$ mixed state with the mixing angle $\phi=-(55 \pm 5)^{\circ}$, can give a decay width consistent with the data $(\Gamma<2.3 \mathrm{MeV})$

$$
\Gamma\left(D_{s 1}(2536)\right) \simeq 0.4 \sim 2.5 \mathrm{MeV} .
$$

In contrast, if $D_{s 1}(2536)$ is a pure ${ }^{1} P_{1}$ state, its decay width will be $59 \mathrm{MeV}$, which is overestimated by a factor of 20 .

We also derive the width ratio

$$
R \equiv \frac{\Gamma\left(D^{*}(2007)^{0} K^{+}\right)}{\Gamma\left(D^{*}(2010)^{+} K^{0}\right)} \simeq 1.2 \sim 1.7
$$

which is consistent with the experimental result, $R=1.27 \pm 0.27$. In Fig. 1(B), the change of the strong decay width $\Gamma\left(D_{s 1}(2536)\right)$ in terms of the mixing angle $\phi$ is presented by treating it as mixed $\left|P_{1}\right\rangle$ state. It should be mentioned that the recent measurements of the angular decomposition of $D_{s 1}(2536)^{+} \rightarrow D^{*+} K_{S}^{0}$ indicate configuration mixings within $D_{s 1}(2536)[78]$.

In the $B$ meson sector, two new narrow excited $B_{1}$ and $B_{s 1}$ mesons are recently reported by CDF, with masses

$$
\begin{array}{r}
M\left(B_{1}\right)=5725 \pm 2 \pm 1 \mathrm{MeV} \\
M\left(B_{s 1}\right)=5829 \pm 1 \mathrm{MeV} .
\end{array}
$$

D0 collaboration also observed the same $B_{1}$ state with a slightly different mass, $M\left(B_{1}\right)=5720 \pm 2.4 \pm 1.4 \mathrm{MeV}$.

The narrowness of these two axial vector states make them good candidates as the narrow heavy partners in the state mixing. $B_{1}$ as a $\left|P_{1}\right\rangle$ state, its strong decay width to $B^{*} \pi$ is predicted to be

$$
\Gamma\left(B_{1}\right) \simeq 30 \mathrm{MeV} .
$$

With the strong decay widths for $B_{2}^{*} \rightarrow B \pi$ and $B^{*} \pi$ calculated, we obtain the strong decay width ratio

$$
R \equiv \frac{\Gamma\left(B_{1}\right)}{\Gamma\left(B_{1}\right)+\Gamma\left(B_{2}^{*}\right)}=0.34
$$

which are in good agreement with the recent D0 data $R=0.477 \pm 0.069 \pm 0.062[10]$.

Note that $B_{J}^{*}(5732)$ in PDG 75] is a broad state with $\Gamma_{\text {exp }}=128 \pm 18 \mathrm{MeV}$. The PDG averaged mass is $5698 \pm 8$ $\mathrm{MeV}$ which makes it lighter than $B_{1}(5725)$. This makes it a natural candidate as the mixed light partner $\left|P_{1}^{\prime}\right\rangle$, for 
which the predicted width is $\Gamma\left(B_{1}^{\prime}\right)=219 \mathrm{MeV}$, this result is compatible with the QCDSR prediction $\Gamma\left(B_{1}^{\prime}\right) \simeq 250$ $\mathrm{MeV}$. As a test, we find that $B_{J}^{*}(5732)$ as a pure ${ }^{3} P_{1}$ state its decay width is $153 \mathrm{MeV}$, which seems to agree well with the PDG suggested value. Whether $B_{J}^{*}(5732)$ is a mixed state $\left|P_{1}^{\prime}\right\rangle$, a pure ${ }^{3} P_{1}$ state, or other configurations, needs further improved experimental measurement.

Similarly, $B_{s 1}$ as a $\left|P_{1}\right\rangle$ state, its strong decay width and decay width ratio to the sum of $B_{s 1}$ and $B_{2 s}^{*}$ widths are

$$
\begin{aligned}
& \Gamma\left(B_{s 1}\right) \simeq 0.4 \sim 1 \mathrm{MeV} \\
& R \equiv \frac{\Gamma\left(B_{s 1}\right)}{\Gamma\left(B_{s 1}\right)+\Gamma\left(B_{2 s}^{*}\right)}=0.02 \sim 0.6 .
\end{aligned}
$$

The predicted width $\Gamma\left(B_{s 1}\right)$ agrees with the data $\Gamma\left(B_{s 1}\right)_{\text {exp }} \sim 1 \mathrm{MeV}[82]$.

Since the mass of $\left|P_{1}^{\prime}\right\rangle$ is slightly lower than that of $\left|P_{1}\right\rangle$, the mass of $B_{s 1}^{\prime}$ (as a $\left|P_{1}^{\prime}\right\rangle$ state) should be less than 5830 $\mathrm{MeV}$. If we assume the mass of $B_{s 1}^{\prime}$ is around $5830 \mathrm{MeV}$, which gives a broad decay width to $B^{*} K$ channel

$$
\Gamma\left(B_{s 1}^{\prime}\right) \simeq 149 \mathrm{MeV}
$$

We should point out that the mass of $B_{s 1}^{\prime}$ is most likely below the threshold of $B^{*} K$, thus, the decay $B_{s 1}^{\prime} \rightarrow B^{*} K$ is kinematically forbidden. In this case the decay width of $B_{s 1}^{\prime}$ will be very narrow. Its decay properties should be similar to those of $D_{s}(2460)$. The isospin violation decay $B_{s 1}^{\prime} \rightarrow B_{s}^{*} \pi$ and radiative decay $B_{s 1}^{\prime} \rightarrow B_{s}^{*} \gamma$ will be the dominant decay modes. A recent study of this scenario was given by Wang with light-cone sum rules [81].

\section{Strong decays of $2 S$ states}
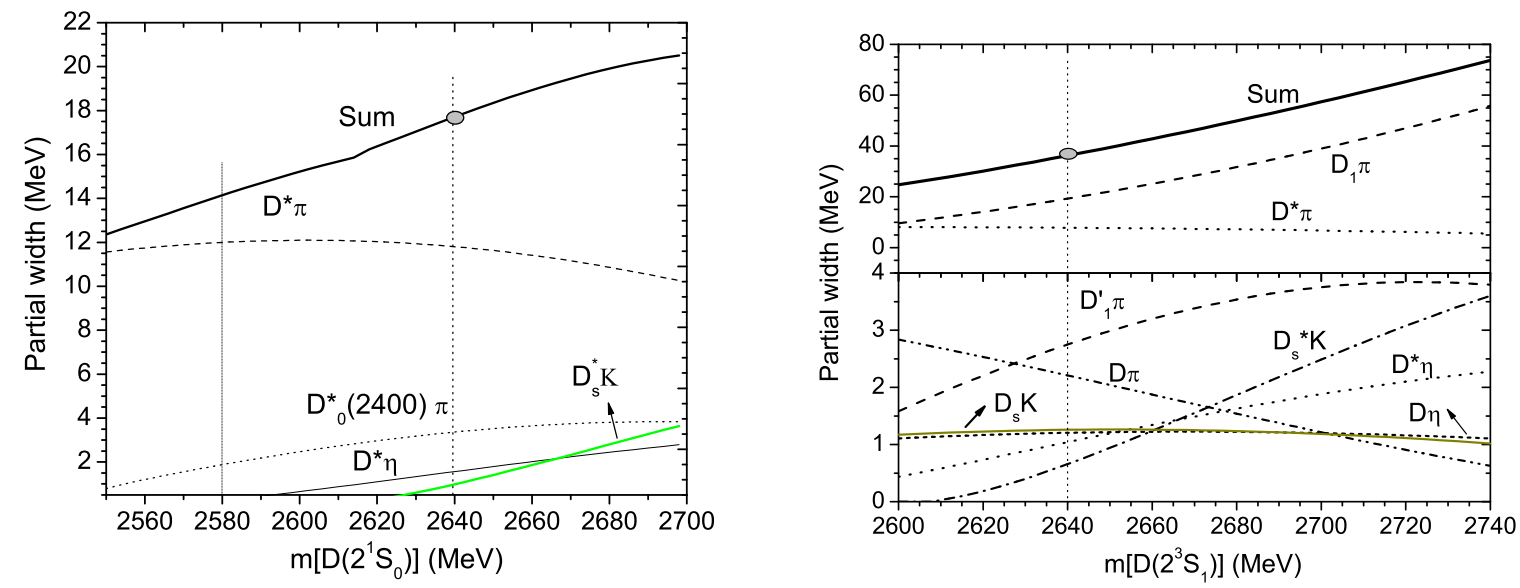

FIG. 2: The partial widths of $D\left(2^{1} S_{0}\right)$ and $D\left(2^{3} S_{1}\right)$ as functions of the mass.

The radially excited heavy-light mesons are still not well-established in experiment, although there are several candidates, such as $D^{*}(2640)^{ \pm}[62], D_{s J}^{*}(2632)[63]$ and $D_{s J}^{*}(2700)[6,6]$. In theory, the radially excited $D$ states $2^{1} S_{0}$ and $2^{3} S_{1}$ were predicted to have masses $\sim 2.58$ and $\sim 2.64 \mathrm{GeV}$, respectively 22], while the radially excited $D_{s}$ states $2^{1} S_{0}$ and $2^{3} S_{1}$ were $\sim 2.6$ and $\sim 2.7 \mathrm{GeV}$, respectively [22, 37]. In this section, we study the strong decays of these excited states into various channels. The mass uncertainties bring uncertainties into the predicted partial decay widths. Occasionally, some of the predicted partial widths exhibit sensitivities to the meson masses. Therefore, we present the strong decay widths of the $D$ and $D_{s}$ radially excited states as functions of their masses within a reasonable range as predicted by theory, and plot them along with their partial decay widths in Figs. 2] and 3, respectively. For a given initial mass, by comparing the relative magnitudes among different partial widths from theoretical prediction and experimental measurement, one can extract additional information about the initial meson quantum numbers. 

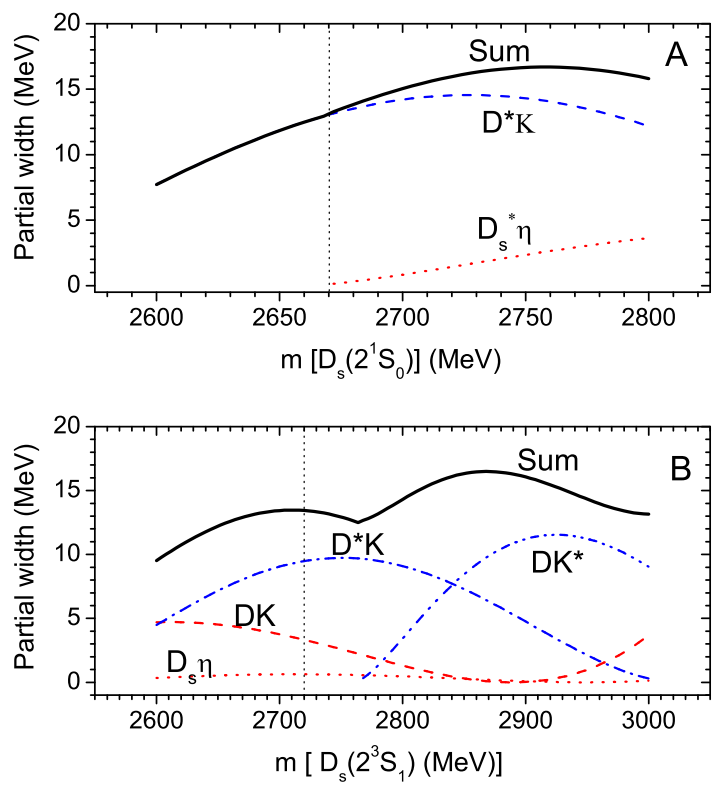

FIG. 3: The partial widths of $D_{s}\left(2^{1} S_{0}\right)$ and $D_{s}\left(2^{3} S_{1}\right)$ as functions of the mass.

\section{Radially excited $D$ mesons}

For a $2{ }^{1} S_{0}$ state with a mass around $2.64 \mathrm{GeV}$, it can decay into $D^{*} \pi, D^{*} \eta, D_{s}^{*} K$ and $D_{0}^{*}(2400) \pi$. In Fig. 2, the partial widths and total strong decay width are shown for a mass range. In these channels, the $D^{*} \pi$ dominates the decays, the total decay width is $\sim 14 \mathrm{MeV}$ at $m\left(D\left(2^{1} S_{0}\right)\right)=2.58 \mathrm{GeV}$, and it shows a flat behavior. Note that the threshold of $D_{s}^{*} K$ channel is very close to $2.64 \mathrm{GeV}$. Some sensitivities to this open channel thus occur in a mass range around $2.6 \mathrm{GeV}$. It shows that this width increases quickly with the masses and will compete again $D^{*} \eta$.

For the radially excited state $2^{3} S_{1}$, its dominant decay channel is $D_{1}(2420) \pi$, while the other partial widths are much smaller (see lower panel of Fig. 22). Again, the $D^{*} \pi$ partial width appears insensitive to the initial $D$ meson mass.

Comparing the decay patterns between $2^{1} S_{0}$ and $2^{3} S_{1}$ in Fig. 2] we find it useful for clarifying $D^{*}(2640)^{ \pm}$. This state was first seen by BELPHI in $D^{*+} \pi^{+} \pi^{-}$channel with a narrow width $<15 \mathrm{MeV}$ [62], but has not yet been confirmed by other experiments. If it is a genuine resonance, it will fit better into the $2^{3} S_{1}$ state instead of $2^{1} S_{0}$ due to its dominant decays into $D^{*+} \pi^{+} \pi^{-}$which can occur via the main channel $D^{*}(2640)^{+} \rightarrow D_{1}(2420)^{0} \pi^{+}$. In contrast, the assignment to a $2^{1} S_{0}$ state will imply a dominant decay channel to $D^{*} \pi$ which is not supported by the data. Although the predicted width $\sim 34 \mathrm{MeV}$ overestimates the data by nearly a factor of two, it should be more urgent to establish it in experiment and have more precise measurement of its partial decay widths to both $D^{*} \pi$ and $D^{*} \pi \pi$.

\section{Radially excited $D_{s}$ mesons}

There are experimental signals for several excited $D_{s}$ states, i.e. $D_{s J}(2632)$ [63], $D_{s J}(2690), D_{s J}(2860)$ [6], and $D_{s J}(2708)\left[\right.$ 7, 8] for which the spectroscopic classification is still unsettled. The $D_{s J}(2690)$ and $D_{s J}(2708)$ are likely to the same state as they have similar masses and both are broad. We shall compare these experimental observations with our model predictions in order to learn more about their spectroscopic classifications.

$D_{s J}(2632)$ was reported by SELEX as a narrow state, i.e. $\Gamma<17 \mathrm{MeV}$, in $D_{s} \eta$ and $D K$ channels [63]. The measured ratio of the partial widths is

$$
R \equiv \frac{\Gamma\left(D^{0} K^{+}\right)}{\Gamma\left(D_{s} \eta\right)}=0.16 \pm 0.06
$$

Its dominant decay into $D_{s} \eta$ makes it difficult to assign it into any simple $c \bar{q}$ scenario [80]. In particular, since a $2^{1} S_{0}$ 
state is forbidden to decay into $D_{s} \eta$ and $D K$, it rules out $D_{s J}(2632)$ to be a radially excited $0^{-}$.

As shown by Fig. 3, the decay of a $2^{3} S_{1}$ state turns to be dominated by $D^{*} K$ and possibly $D K$, while its decay into $D_{s} \eta$ is rather small. Therefore, a simple $2^{3} S_{1}$ cannot explain its decay pattern as well. Some more investigations of the nature of $D_{s J}(2632)$ can be found in the literature, and here we restrict our attention on the output of our model calculations.

$D_{s J}^{*}(2860)$ and $D_{s J}^{*}(2690)$ from BaBar [6] (or $D_{s J}(2708)$ from Belle [7, 8]) have widths

$$
\begin{gathered}
\Gamma\left(D_{s J}(2860)\right)=48 \pm 17 \mathrm{MeV}, \\
\Gamma\left(D_{s J}(2690)\right)=112 \pm 43 \mathrm{MeV},
\end{gathered}
$$

and both are observed in the $D K$ channel, and no evidences are seen in $D^{*} K$ and $D_{s} \eta$ modes. Compare these with Fig. 3, it shows that neither of them can easily fit in $2^{1} S_{0}$ or $2^{3} S_{1}$.

By fixing the masses of $2^{1} S_{0}$ and $2^{3} S_{1}$ states as suggested by the quark model [37], i.e. $m\left(D_{s}\left(2^{1} S_{0}\right)\right)=2.64 \mathrm{GeV}$ and $m\left(D_{s}\left(2^{3} S_{1}\right)\right)=2.71 \mathrm{GeV}$, we obtain their strong decay widths

$$
\begin{aligned}
\Gamma\left(D_{s}\left(2^{1} S_{0}\right)\right) & \simeq 11 \mathrm{MeV}, \\
\Gamma\left(D_{s}\left(2^{3} S_{1}\right)\right) & \simeq 14 \mathrm{MeV},
\end{aligned}
$$

which turn out to be narrow. For $D_{s}\left(2{ }^{1} S_{0}\right)$, the predicted dominant decay mode is $D^{*} K$, while the $D K$ channel is forbidden. For $D_{s}\left(2^{3} S_{1}\right)$, there are two main decay channels $D^{*} K$ and $D K$, and they give a ratio of

$$
R \equiv \frac{\Gamma\left(D^{*} K\right)}{\Gamma(D K)} \simeq 2.6
$$

The $D_{s} \eta$ channel is also opened, but is negligibly small in comparison with $D K$ and $D^{*} K$.

As $D_{s J}^{*}(2860)$ has a relatively larger mass to fit in a $D$-wave state, we shall examine it with $D$-wave decays in the following subsection.
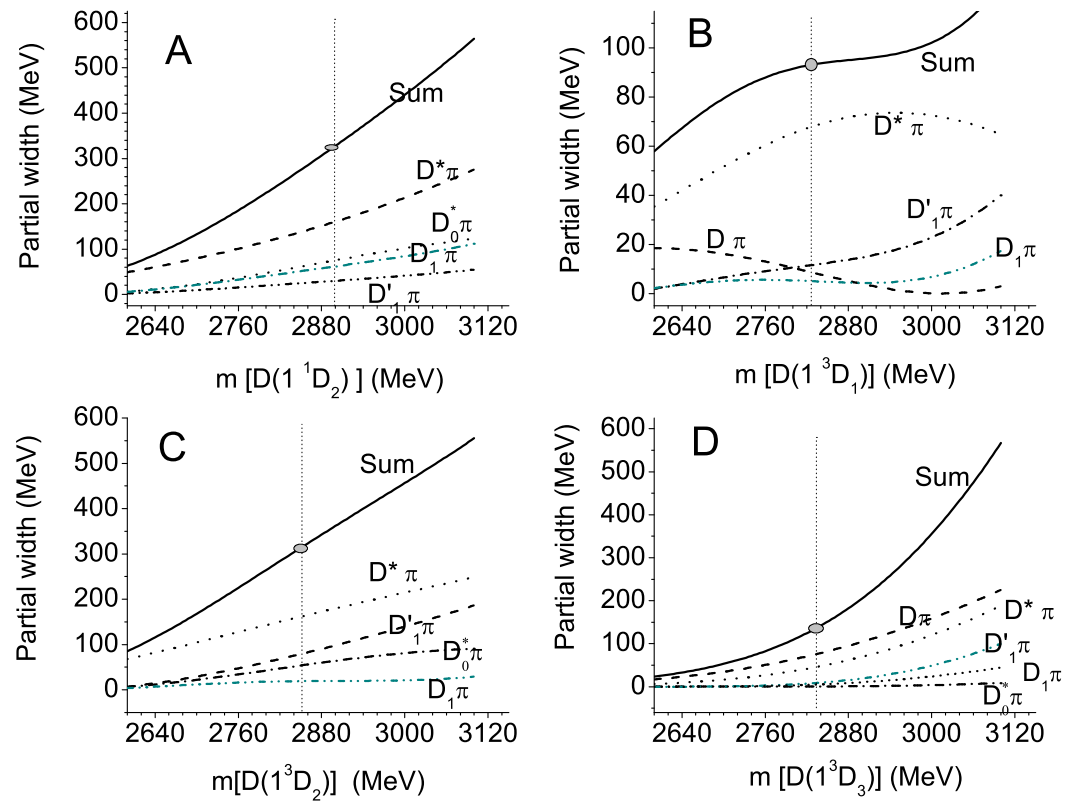

FIG. 4: The partial widths of $D\left(1^{1} D_{2}\right), D\left(1^{3} D_{1}\right), D\left(1^{3} D_{2}\right)$ and $D\left(1^{3} D_{3}\right)$ as functions of the mass. 

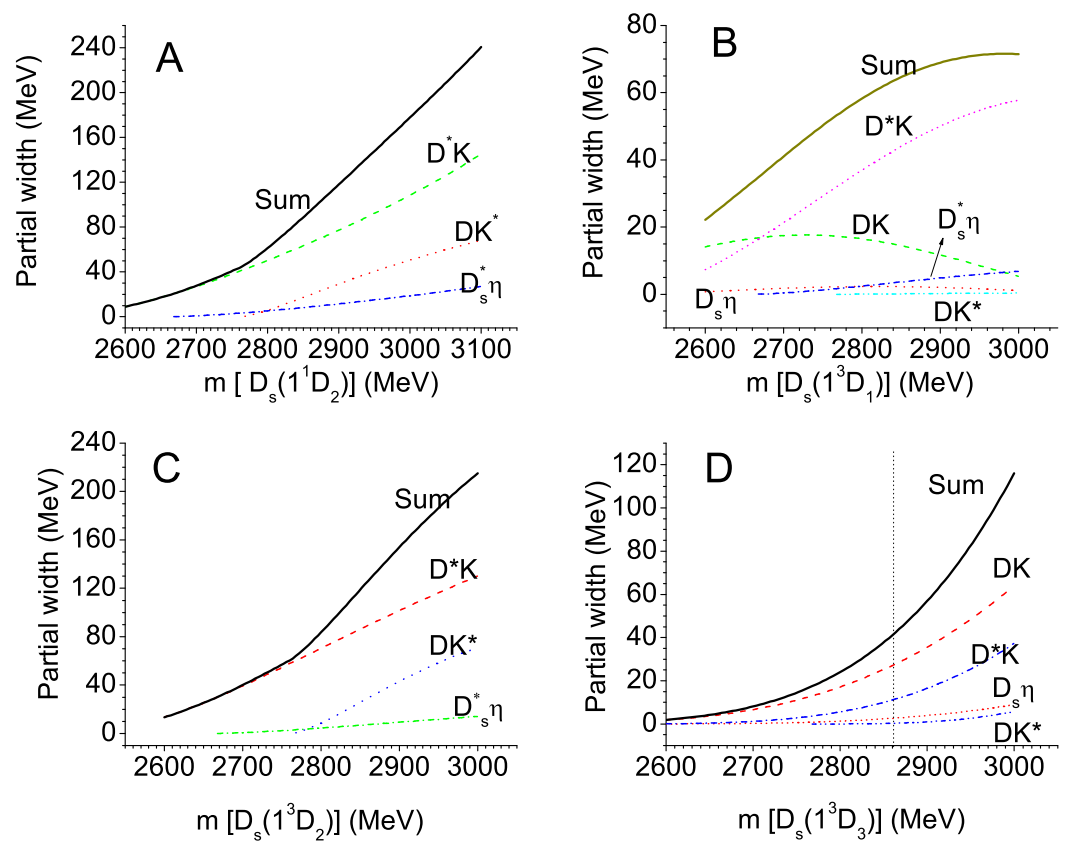

FIG. 5: The partial widths of $D_{s}\left(1^{1} D_{2}\right), D_{s}\left(1^{3} D_{1}\right), D_{s}\left(1^{3} D_{2}\right)$ and $D_{s}\left(1^{3} D_{3}\right)$ as functions of the mass. When we calculate the partial width of the $D K^{*}$ channel, the $D$ meson is looked as the emitted pseudoscalar meson in the $S U(4)$ case 74 ].

\section{Strong decays of $1 D$ states}

Theoretical predicted masses of the $D$-wave excited $D$ and $D_{s}$ mesons are in a range of $2.8 \sim 2.9 \mathrm{GeV}$ [22, 47]. To see the decay properties of those $D$-wave states, we plot their main decay partial widths in their possible mass region in Figs. 4 and 5 for $D$ and $D_{s}$, respectively.

\section{Excited D mesons}

In Fig. 4. decays of four $D$ wave states are presented. It shows that the widths of $D\left(1^{1} D_{2}\right)$ and $D\left(1^{3} D_{2}\right)$ states are dominated by $D^{*} \pi$ decay while the $D \pi / D \eta$ channels are forbidden. At $\sim 2.8 \mathrm{GeV}$, they have very broad widths larger than $300 \mathrm{MeV}$. As $D^{*}$ dominantly decays into $D \pi$, such broad widths imply that their dominant final states are $D \pi \pi$, and it might be difficult to identify them in experiment. This may explain why these states are still evading experimental observations.

For $D\left(1^{3} D_{1}\right), D^{*} \pi$ is also the main decay channel, but with a much smaller width. In contrast, $D \pi$ dominates the decay of $D\left(1^{3} D_{3}\right)$. With theory-suggested masses $m\left(D\left(1^{3} D_{1}\right)\right)=2.82$ and $m\left(D\left(1^{3} D_{3}\right)\right)=2.83$ [22], the total pionic decay widths for $D\left(1^{3} D_{1}\right)$ and $D\left(1^{3} D_{3}\right)$ are predicted to be

$$
\begin{aligned}
\Gamma\left(D\left(1^{3} D_{1}\right)\right) & \simeq 93 \mathrm{MeV}, \\
\Gamma\left(D\left(1^{3} D_{3}\right)\right) & \simeq 130 \mathrm{MeV},
\end{aligned}
$$

and the predicted ratios between the $D^{*} \pi$ and $D \pi$ widths are

$$
\begin{gathered}
R\left(D\left(1^{3} D_{1}\right)\right) \equiv \frac{\Gamma(D \pi)}{\Gamma\left(D^{*} \pi\right)} \simeq 0.12, \\
R\left(D\left(1^{3} D_{3}\right)\right) \equiv \frac{\Gamma(D \pi)}{\Gamma\left(D^{*} \pi\right)} \simeq 1.7 .
\end{gathered}
$$

For $D\left(1^{3} D_{3}\right)$, the dominance of $D \pi$ decay suggests that it is relatively more accessible in experiment. 


\section{Excited $D_{s}$ mesons}

For $D_{s}\left(1^{1} D_{2}\right)$ and $D_{s}\left(1^{3} D_{2}\right)$, three important partial widths of $D^{*} K, D K^{*}$ and $D_{s}^{*} \eta$ are presented in Fig. 5 , and both states are dominated by the $D^{*} K$ decay. It is interesting to see that the mass of $\sim 2.8 \mathrm{GeV}$ is about the threshold for $D K^{*}$. Although this decay mode is negligible near threshold, it can become important in case that the masses for these two $D$-wave states are above $2.8 \mathrm{GeV}$.

At $2.8 \mathrm{GeV}$, the total widths are dominated by the $D^{*} K$ mode, which are

$$
\begin{aligned}
\Gamma\left(D_{s}\left(1^{1} D_{2}\right)\right) & \simeq 61 \mathrm{MeV} \\
\Gamma\left(D_{s}\left(1^{3} D_{2}\right)\right) & \simeq 84 \mathrm{MeV}
\end{aligned}
$$

These results can guide a search for these two states around $2.8 \mathrm{GeV}$.

As shown in Fig. 5. $D^{*} K$ and $D K$ are two dominant decay modes for $D_{s}\left(1^{3} D_{1}\right)$ if its mass is around $2.8 \mathrm{GeV}$, and the predicted width is relatively narrow. Implications from such an assignment will be discussed in subsection $\mathrm{VE}$.

Compared with the $D_{s}\left(1^{3} D_{1}\right)$ decay, the dominant decay mode of $D_{s}\left(1^{3} D_{3}\right)$ state is $D K$ around $2.8 \mathrm{GeV}$. With a higher mass the $D^{*} K$ channel becomes increasingly important. This feature fits well the experimental observation for $D_{s J}^{*}(2860)$, and makes it a possible assignment for this state.

To be more specific, $D_{s J}^{*}(2860)$ as a $D_{s}\left(1^{3} D_{3}\right)$ state, its predicted width is

$$
\Gamma\left(D_{s}\left(1^{3} D_{3}\right)\right) \simeq 41 \mathrm{MeV}
$$

and the dominant decay mode is $D K$. In comparison, the decays via $D K^{*}$ and $D_{s} \eta$ are much less important (see the Fig. [5] and Tab. VIII). The ratio of $D K$ and $D^{*} K$ is found to be

$$
R\left(D_{s}\left(1^{3} D_{3}\right)\right) \equiv \frac{\Gamma(D K)}{\Gamma\left(D^{*} K\right)} \simeq 2.3,
$$

which is also consistent with the experiment [6]. This assignment agrees with results of Refs. 35, 43, 44].

Some models also suggested that $D_{s J}^{*}(2860)$ could be a $2^{3} P_{0}$ state [34, 35, 37], for which only decay mode $D K$ and $D_{s} \eta$ are allowed. In our model, a $2^{3} P_{0}$ state leads to decay amplitude

$$
\begin{aligned}
& \mathcal{M}\left(2^{3} P_{0} \rightarrow\left|1^{1} S_{0}\right\rangle \mathbb{P}\right) \\
= & i \frac{1}{4} \sqrt{\frac{1}{15}} g_{I} F \frac{q^{\prime}}{\alpha}\left[g_{10}^{z} \mathcal{G} q \frac{q^{\prime 2}}{\alpha^{2}}-\frac{1}{3} g_{10}^{z} h q^{\prime}\left(1+\frac{q^{\prime 2}}{2 \alpha^{2}}\right)\right. \\
& \left.-2 \sqrt{2} g_{10}^{+} h q^{\prime}\left(7-\frac{q^{\prime 2}}{\alpha^{2}}\right)\right]
\end{aligned}
$$

with which its partial decay width to $D K$ is about $\Gamma=184 \mathrm{MeV}$, and much broader than the experimental observation.

\section{E. The $2^{3} S_{1}-1^{3} D_{1}$ mixing}

In Ref. [37], a mixing scheme between $2^{3} S_{1}$ and $1^{3} D_{1}$ was proposed as a solution for the relatively broad $D_{s J}^{*}(2690)$, i.e.

$$
\begin{aligned}
\left|D_{s 1}^{*}(2690)\right\rangle & =\sin (\phi)\left|1^{3} D_{1}\right\rangle+\cos (\phi)\left|2^{3} S_{1}\right\rangle, \\
\left|D_{s 1}^{*}(2810)\right\rangle & =\cos (\phi)\left|1^{3} D_{1}\right\rangle-\sin (\phi)\left|2^{3} S_{1}\right\rangle
\end{aligned}
$$

where an orthogonal state $D_{s 1}^{*}(2810)$ was also predicted. The mixing angle was found to favor $\phi=-0.5$ radians, i.e. $\phi \simeq-27^{\circ}$. According to such a mixing scheme, $D_{s 1}^{*}(2810)$ will also be a broad state and dominated by $1^{3} D_{1}$ configuration.

Taking the mixing scheme of Eq. (63), we plot the widths of $D_{s J}^{*}(2690)$ and $D_{s J}^{*}(2810)$ in terms of mixing angle $\phi$ in Fig. 6. From the figure, it shows that if we take the mixing angle $\phi \simeq-27^{\circ}$ as predicted in Ref. [37], the decay modes of $D_{s J}^{*}(2690)$ is dominated by $D^{*} K$, which disagrees with the experimental observation. Nevertheless, the predicted decay width of $D_{s J}^{*}(2690), \Gamma \sim 25 \mathrm{MeV}$, is underestimated by at least a factor of 2 compared with the data. If the $2 S-1 D$ mixing is small, e.g. $D_{s J}^{*}(2690)$ is a pure $1^{3} D_{1}$ state, the predicted decay width is $\Gamma \sim 42 \mathrm{MeV}$, which is close to the lower limit of the data. However, the ratio $R=\Gamma(D K) / \Gamma\left(D^{*} K\right) \sim 0.8$ disagrees with the observation that the $D K$ channel dominates the decay modes. 


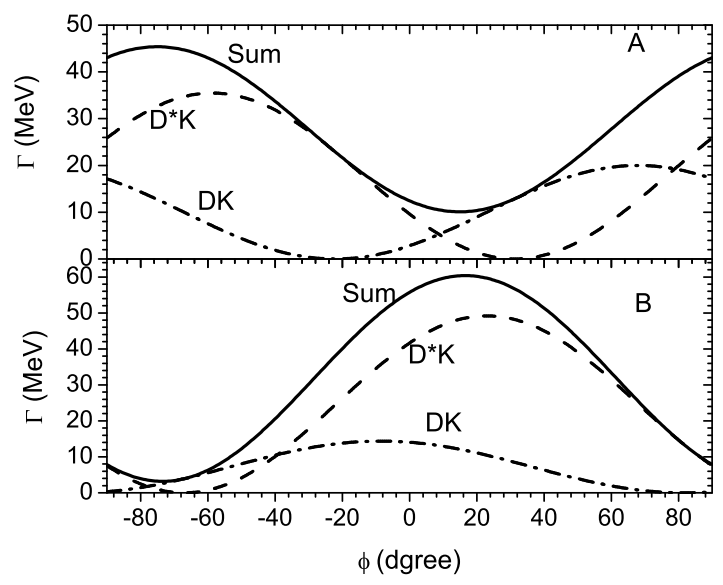

FIG. 6: The partial widths of low vector (A) and high vector (B) as functions of the mixing angle $\phi$.

If we set $\phi \simeq 30^{\circ}$, which implies that the sign of the spin-orbit splitting term is now negative to keep the correct mass ordering, the $D^{*} K$ channel in $D_{s J}^{*}(2690)$ decay will be completely suppressed. This is consistent with the observations except that the decay width $\Gamma \sim 15 \mathrm{MeV}$ is too small to compare with the data.

For the high vector $D_{s J}^{*}(2810)$, with $\phi \simeq \pm 30^{\circ}$, its width reads $\sim 40-60 \mathrm{MeV}$ and is dominated by the $D^{*} K$. Meanwhile, its branching ratio to $D K$ is still sizeable.

Our test of the property of $D_{s J}^{*}(2690)$ in the $2 S-1 D$ mixing scenario does not fit in the data very well. To clarify the nature of $D_{s J}^{*}(2690)$, more accurate measurements of its width and ratio $R=D K / D^{*} K$, and experimental search for the accompanying $D_{s J}^{*}(2810)$ in the $D K$ and $D^{*} K$ channels are needed.

\section{SUMMARY}

In the chiral quark model framework, we systematically study the strong decays of heavy-light mesons in $\mathbb{M} \rightarrow$ $\left|1^{1} S_{0}\right\rangle \mathbb{P}, \mathbb{M} \rightarrow\left|1^{3} S_{1}\right\rangle \mathbb{P}, \mathbb{M} \rightarrow\left|1^{3} P_{0}\right\rangle \mathbb{P}, \mathbb{M} \rightarrow\left|1^{1} P_{1}\right\rangle \mathbb{P}$, and $\mathbb{M} \rightarrow\left|1^{3} P_{1}\right\rangle \mathbb{P}$. By adopting commonly used values for the constituent quark masses and pseudoscalar meson decay constants, we make a full analysis of the strong decays of all the excited $D^{*}, D_{s}^{*}, B^{*}$ and $B_{s}^{*}$, and find that most available data can be consistently explained in this framework. We summarize our major results as follows.

\section{A. Excited $D$ mesons}

The calculated partial decay widths for the $D^{*}\left(1^{3} S_{1}\right), D_{0}^{*}(2400)$ as a $1^{3} P_{0}$ state and $D_{2}^{*}(2460)$ as a $1^{3} P_{2}$ state are in good agreement with the data in our model and support their assignments as the low-lying excited $D^{*}$.

State mixing between the $1^{1} P_{1}$ and $1^{3} P_{1}$ is favored. With the mixing angle, $\phi \simeq-(55 \pm 5)^{\circ}$, which is consistent with the prediction of Ref. 23], the narrow $D_{1}(2420)$ and broad $D_{1}^{\prime}(2430)$ can be well explained as mixing states between $1^{1} P_{1}$ and $1^{3} P_{1}$. Precise measurement of the mass of the broad $D_{1}^{\prime}(2430)$ is needed.

Our result shows that assigning $D^{*}(2640)$ to be a radially excited $2^{3} S_{1}$ state can naturally explain its observation in $D^{*+} \pi^{+} \pi^{-}$final state, although the predicted width $\sim 34 \mathrm{MeV}$ still possesses some discrepancies with the data. A search for the $D^{*}(2640)$ in the $D_{1}(2420) \pi$ channel is strongly recommended.

Although the $2 S$ and $1 D$ excited $D$ states are still not well established, we analyzed their partial decay widths in their possible mass regions, which should be useful for future experimental studies. The decay widths of $2 S$ states turn to be narrow, namely, at the order of a few tens of $\mathrm{MeV}$. Our results shows that $D\left(2{ }^{1} S_{0}\right)$ is dominated by the $D^{*} \pi$ decay channel, while both $D^{*} \pi$ and $D_{1}(2420) \pi$ are important for $D\left(2^{3} S_{1}\right)$. Both $D\left(1^{1} D_{2}\right)$ and $D\left(1^{3} D_{2}\right)$ have very broad widths $\gtrsim 300 \mathrm{MeV}$, which may be difficult to identify in experiment. The decay widths of $D\left(1^{3} D_{1}\right)$ and $D\left(1^{3} D_{3}\right)$ are $\gtrsim 100 \mathrm{MeV}$. The former has dominant decays into $D^{*} \pi$ and sizeable widths to $D \pi$ as well. For $D\left(1^{3} D_{3}\right)$, both $D \pi$ and $D^{*} \pi$ are important. 


\section{B. Excited $D_{s}$ mesons}

$D_{s}(2460)$ and $D_{s 1}(2536)$ are identified as partners due to mixing between ${ }^{1} P_{1}$ and ${ }^{3} P_{1}$. With mixing angle $\phi \simeq$ $-(55 \pm 5)^{\circ}$, our predictions for $D_{s 1}(2536)$ are in good agreement with the data. Note that $D_{s}(2460)$ is below the strong decay threshold.

$D_{s 2}^{*}(2573)$ is consistent with a $1^{3} P_{2}$ state. Its partial decay width to $D K$ is dominant while to $D^{*} K$ is very small $(\sim 1 \mathrm{MeV})$. This explains its absence in $D^{*} K$ channel 75 .

For those not-well-established states, by analyzing their decay properties, we find that $D_{s J}^{*}(2860)$ strongly favors a $D_{s}\left(1^{3} D_{3}\right)$ state, while the $D_{s J}^{*}(2632)$ and $D_{s J}^{*}(2690)$ cannot fit in any pure $D_{s}^{*}$ configurations.

For the unobserved $2 S$ states, $D_{s}\left(2^{1} S_{0}\right)$ and $D_{s}\left(2^{3} S_{1}\right)$, their decay widths are predicted to be an order of $\sim 15$ $\mathrm{MeV}$, and dominated by the $D^{*} K$ decay mode.

For the unobserved $1 D$ states, $D_{s}\left(1^{1} D_{2}\right), D_{s}\left(1^{3} D_{2}\right)$ and $D_{s}\left(1^{3} D_{1}\right)$, their decay widths are of the order of $60 \sim 80$ $\mathrm{MeV}$ at a mass of $\sim 2.8 \mathrm{GeV}$. The $D_{s}\left(1^{1} D_{2}\right)$ and $D_{s}\left(1^{3} D_{2}\right)$ decay are dominated by the $D^{*} K$ mode, while $D_{s}\left(1^{3} D_{1}\right)$ is dominated by the $D^{*} K$ and $D K$ together.

\section{Excited $B$ mesons}

We also study the decay properties of the newly observed bottom states $B_{1}(5725), B_{2}^{*}(5829)$. Our calculations strongly suggest that $B_{1}(5725)$ is a mixed $\left|P_{1}\right\rangle$ state, and $B_{2}^{*}(5829)$ satisfies an assignment of $1^{3} P_{2}$.

The $B_{J}^{*}(5732)$, which was first reported by L3 collaboration, can be naturally explained as the broad partner $\left(\left|P_{1}^{\prime}\right\rangle\right)$ of $B_{1}(5725)$ in the ${ }^{1} P_{1}$ and ${ }^{3} P_{1}$ mixing scheme. Its predicted width is $\Gamma\left(B_{1}^{\prime}\right)=219 \mathrm{MeV}$, which is larger than the PDG suggested value $\Gamma_{\exp }=128 \pm 18 \mathrm{MeV}$. In contrast, as a pure $1^{3} P_{1}$ state, its decay width is $\Gamma\left(B_{1}^{\prime}\right)=153 \mathrm{MeV}$. Whether $B_{J}^{*}(5732)$ is a mixed state $\left|P_{1}^{\prime}\right\rangle$, a pure $1^{3} P_{1}$ state, or other configurations, should be further studied.

The theoretical prediction of the mass for $B_{0}^{*}\left({ }^{3} P_{0}\right)$ meson is about $5730 \mathrm{MeV}$. Its decay into $B \pi$ has a broad width $\Gamma\left(B_{0}^{*}\right)=272 \mathrm{MeV}$ according to our prediction.

\section{Excited $B_{s}$ mesons}

The two new narrow bottom-strange mesons $B_{s 1}(5830)$ and $B_{s 2}^{*}(5839)$ observed by CDF are likely the mixed state $P_{1}$ and the $1^{3} P_{2}$ state, respectively, though their decay widths and ratios are not given. $B_{s 1}(5830)$ as a $\left|P_{1}\right\rangle$ state, its predicted width and decay ratio are $\Gamma\left(B_{s 1}\right) \simeq(0.4 \sim 1) \mathrm{MeV}$ and $\Gamma\left(B_{s 1}\right) /\left(\Gamma\left(B_{s 1}\right)+\Gamma\left(B_{2 s}^{*}\right)\right)=0.02 \sim 0.6 . B_{s}(5839)$ as the ${ }^{3} P_{2}$ state, its decay width and width ratio predicted by us are $\Gamma\left(B_{s 2}^{*}\right) \simeq 2 \mathrm{MeV}$ and $\Gamma\left(B^{*} K\right) / \Gamma(B K) \simeq 6 \%$.

The theoretical predictions for the $B_{s 0}^{*}$ masses is about $5800 \mathrm{MeV}$. In our model it has a broad width $\Gamma\left(B_{0}^{*}\right)=227$ $\mathrm{MeV}$. For $B_{s 1}^{\prime}$ if its mass is above the threshold of $B^{*} K$, it will be a broad state with $\Gamma\left(B_{s 1}^{\prime}\right) \simeq 149 \mathrm{MeV}$. Otherwise, it should be a narrow state.

It should be mentioned that uncertainties with quark model parameters can give rise to uncertainties with the theoretical results. A qualitative examination shows that such uncertainties can be as large as $10-20 \%$, which are a typical order for quark model approaches. Interestingly, systematics arising from such a simple prescription are useful for us to gain insights into the effective degrees of freedom inside those heavy-light mesons and the underlying dynamics for their strong decays.

\section{Acknowledgements}

The authors wish to thank F.E. Close and S.-L. Zhu for useful discussions. This work is supported, in part, by the National Natural Science Foundation of China (Grants 10675131 and 10775145), Chinese Academy of Sciences (KJCX3-SYW-N2), the U.K. EPSRC (Grant No. GR/S99433/01), the Post-Doctoral Programme Foundation of China, and K. C. Wong Education Foundation, Hong Kong.

[1] B. Aubert et al. [BABAR Collaboration], Phys. Rev. Lett. 90, 242001 (2003) arXiv:hep-ex/0304021].

[2] B. Aubert et al. [BABAR Collaboration], Phys. Rev. D 69, 031101 (2004) arXiv:hep-ex/0310050.

[3] D. Besson et al. [CLEO Collaboration], Phys. Rev. D 68, 032002 (2003) [Erratum-ibid. D 75, 119908 (2007)] arXiv:hep-ex/0305100. 
[4] K. Abe et al., Phys. Rev. Lett. 92, 012002 (2004) arXiv:hep-ex/0307052.

[5] P. Krokovny et al. [Belle Collaboration], Phys. Rev. Lett. 91, 262002 (2003) arXiv:hep-ex/0308019.

[6] B. Aubert [BABAR Collaboration], Phys. Rev. Lett. 97, 222001 (2006) arXiv:hep-ex/0607082.

[7] J. Brodzicka et al. [Belle Collaboration], arXiv:0707.3491 [hep-ex].

[8] K. Abe et al. [Belle Collaboration], arXiv:hep-ex/0608031

[9] CDF Collaboration, "Study of orbitally $(L=1) B$ mesons", CDF Note 8945 (2007).

[10] V. M. Abazov et al. [D0 Collaboration], Phys. Rev. Lett. 99, 172001 (2007)

[11] et al. [CDF Collaboration], arXiv:0710.4199 [hep-ex].

[12] et al. [D0 Collaboration], arXiv:0711.0319 [hep-ex].

[13] S. Bianco, AIP Conf. Proc. 814, 24 (2006) arXiv:hep-ex/0512073.

[14] I. Kravchenko [CDF Collaboration], In the Proceedings of 4th Flavor Physics and CP Violation Conference (FPCP 2006), Vancouver, British Columbia, Canada, 9-12 Apr 2006, pp 016 arXiv:hep-ex/0605076.

[15] R. Waldi, arXiv:hep-ex/0703043.

[16] A. Zghiche [BaBar Collaboration], arXiv:0710.0314 [hep-ex].

[17] V. Poireau, arXiv:0711.3683 [hep-ex].

[18] R. K. Mommsen, Nucl. Phys. Proc. Suppl. 170, 172 (2007) arXiv:hep-ex/0612003.

[19] M. Kreps [CDF Collaboration], arXiv:0711.4890 [hep-ex].

[20] F. E. Close and E. S. Swanson, Phys. Rev. D 72, 094004 (2005) arXiv:hep-ph/0505206.

[21] S. Godfrey, Phys. Rev. D 72, 054029 (2005) arXiv:hep-ph/0508078.

[22] S. Godfrey and N. Isgur, Phys. Rev. D 32, 189 (1985).

[23] S. Godfrey and R. Kokoski, Phys. Rev. D 43, 1679 (1991).

[24] N. Isgur and M. B. Wise, Phys. Rev. Lett. 66, 1130 (1991).

[25] M. Di Pierro and E. Eichten, Phys. Rev. D 64, 114004 (2001) arXiv:hep-ph/0104208.

[26] E. J. Eichten, C. T. Hill and C. Quigg, Phys. Rev. Lett. 71, 4116 (1993) arXiv:hep-ph/9308337.

[27] A. F. Falk and T. Mehen, Phys. Rev. D 53, 231 (1996) arXiv:hep-ph/9507311.

[28] K. O. E. Henriksson, T. A. Lahde, C. J. Nyfalt and D. O. Riska, Nucl. Phys. A 686, 355 (2001) arXiv:hep-ph/0009095.

[29] N. Tregoures and W. Roberts, Phys. Rev. D 61, 074032 (2000) arXiv:hep-ph/9911211.

[30] J. L. Goity and W. Roberts, Phys. Rev. D 60, 034001 (1999) arXiv:hep-ph/9809312 .

[31] S. L. Zhu and Y. B. Dai, Mod. Phys. Lett. A 14, 2367 (1999) arXiv:hep-ph/9811449.

[32] Y. B. Dai and S. L. Zhu, Phys. Rev. D 58, 074009 (1998) arXiv:hep-ph/9802224.

[33] A. H. Orsland and H. Hogaasen, Eur. Phys. J. C 9, 503 (1999) arXiv:hep-ph/9812347].

[34] E. Van Beveren and G. Rupp, Phys. Rev. Lett. 97, 202001 (2006) arXiv:hep-ph/0606110.

[35] B. Zhang, X. Liu, W. Z. Deng and S. L. Zhu, Eur. Phys. J. C 50, 617 (2007) arXiv:hep-ph/0609013].

[36] W. Wei, X. Liu and S. L. Zhu, Phys. Rev. D 75, 014013 (2007) arXiv:hep-ph/0612066.

[37] F. E. Close, C. E. Thomas, O. Lakhina and E. S. Swanson, Phys. Lett. B 647, 159 (2007) arXiv:hep-ph/0608139].

[38] P. Colangelo, F. De Fazio, S. Nicotri and M. Rizzi, Phys. Rev. D 77, 014012 (2008) arXiv:0710.3068 [hep-ph]].

[39] Z. G. Wang, arXiv:0708.0155 [hep-ph].

[40] F. K. Guo, P. N. Shen and H. C. Chiang, Phys. Lett. B 647, 133 (2007) arXiv:hep-ph/0610008.

[41] A. Faessler, T. Gutsche, V. E. Lyubovitskij and Y. L. Ma, arXiv:0801.2232 [hep-ph].

[42] S. Yasui and M. Oka, Phys. Rev. D 76, 034009 (2007) arXiv:0704.1345 [hep-ph]].

[43] P. Colangelo, F. De Fazio and S. Nicotri, Phys. Lett. B 642, 48 (2006) arXiv:hep-ph/0607245.

[44] J. Koponen [UKQCD Collaboration], arXiv:0708.2807 [hep-lat].

[45] J. Vijande, F. Fernandez and A. Valcarce, J. Phys. G 31, 481 (2005) arXiv:hep-ph/0411299.

[46] J. Vijande, F. Fernandez and A. Valcarce, Phys. Rev. D 73, 034002 (2006) [Erratum-ibid. D 74, 059903 (2006)] arXiv:hep-ph/0601143.

[47] T. Matsuki, T. Morii and K. Sudoh, Prog. Theor. Phys. 117, 1077 (2007) arXiv:hep-ph/0605019.

[48] J. Vijande, A. Valcarce and F. Fernandez, Phys. Rev. D 77, 017501 (2008) arXiv:0711.2359 [hep-ph]].

[49] D. M. Li, B. Ma and Y. H. Liu, Eur. Phys. J. C 51, 359 (2007) arXiv:hep-ph/0703278.

[50] E. S. Swanson, AIP Conf. Proc. 892, 362 (2007) [J. Phys. Conf. Ser. 69, 012007 (2007)].

[51] D. Ebert, V. O. Galkin and R. N. Faustov, Phys. Rev. D 57, 5663 (1998) [Erratum-ibid. D 59, 019902 (1999)] arXiv:hep-ph/9712318.

[52] T. Barnes, AIP Conf. Proc. 814, 735 (2006) arXiv:hep-ph/0510365.

[53] E. Swanson, AIP Conf. Proc. 814, 203 (2006) [Int. J. Mod. Phys. A 21, 733 (2006)] arXiv:hep-ph/0509327.

[54] E. S. Swanson, Phys. Rept. 429, 243 (2006) arXiv:hep-ph/0601110].

[55] J. L. Rosner, J. Phys. G 34, S127 (2007) arXiv:hep-ph/0609195].

[56] S. L. Zhu, arXiv:0707.2623 [hep-ph].

[57] J. L. Rosner, J. Phys. Conf. Ser. 69, 012002 (2007) arXiv:hep-ph/0612332.

[58] S. Nicotri, arXiv:0711.4756 [hep-ph].

[59] E. Klempt and A. Zaitsev, Phys. Rept. 454, 1 (2007) arXiv:0708.4016 [hep-ph]].

[60] P. Colangelo, F. De Fazio, R. Ferrandes and S. Nicotri, Prog. Theor. Phys. Suppl. 168, 202 (2007) arXiv:0706.0053 [hep-ph]].

[61] B. Ananthanarayan, S. Banerjee, K. Shivaraj and A. Upadhyay, Phys. Lett. B 651, 124 (2007) [arXiv:0706.0942 [hep-ph]].

[62] P. Abreu et al. [DELPHI Collaboration], Phys. Lett. B 426, 231 (1998).

[63] A. V. Evdokimov et al. [SELEX Collaboration], Phys. Rev. Lett. 93 (2004) 242001 arXiv:hep-ex/0406045. 
[64] A. Manohar and H. Georgi, Nucl. Phys. B 234, 189 (1984).

[65] T. Abdullah and F. E. Close, Phys. Rev. D 5, 2332 (1972).

[66] F. E. Close and Z. P. Li, Phys. Rev. D 42, 2194 (1990).

[67] Z. P. Li, Phys. Rev. D 48, 3070 (1993); 50, 5639 (1994); 52, 4961 (1995); Phys. Rev. C 52, 1648 (1995).

68] Z. P. Li, H. X. Ye and M. H. Lu, Phys. Rev. C 56, 1099 (1997) arXiv:nucl-th/9706010.

[69] Q. Zhao, J. S. Al-Khalili and C. Bennhold, Phys. Rev. C 64, 052201 (2001) arXiv:nucl-th/0102043.

[70] Q. Zhao, J. S. Al-Khalili, Z. P. Li and R. L. Workman, Phys. Rev. C 65, 065204 (2002) arXiv:nucl-th/0202067.

[71] Q. Zhao, B. Saghai and Z. P. Li, J. Phys. G 28, 1293 (2002) arXiv:nucl-th/0011069.

[72] Q. Zhao, Z. P. Li and C. Bennhold, Phys. Rev. C 58, 2393 (1998) arXiv:nucl-th/9806100; Phys. Lett. B 436, 42 (1998) arXiv:nucl-th/9803015.

[73] X. H. Zhong, Q. Zhao, J. He and B. Saghai, Phys. Rev. C 76, 065205 (2007) arXiv:0706.3543 [nucl-th]].

[74] X. H. Zhong and Q. Zhao, Phys. Rev. D 77, 074008 (2008).

[75] Particle Data Group, Y. M. Yao et al., J. Phys. G 33, 1 (2006).

[76] K. Abe et al. [Belle Collaboration], Phys. Rev. D 69, 112002 (2004).

[77] J.M. Link et al. [FOCUS Collaboration], Phys. Lett. B586, 11 (2004).

[78] V. Balagura et al. [Belle Collaboration], Phys. Rev. D 77, 032001 (2008) arXiv:0709.4184 [hep-ex]].

[79] K. Abe et al. [Belle Collaboration], Phys. Rev. D 69, 112002 (2004) arXiv:hep-ex/0307021.

[80] T. Barnes, F.E. Close, J.J. Dudek, S. Godfrey, and E.S. Swanson, Phys. Lett. B 600, 223 (2004).

[81] Z. G. Wang, arXiv:0803.1223 [hep-ph].

[82] R. Akers et al. [OPAL Collaboration], Z. Phys. C 66, 19 (1995). 